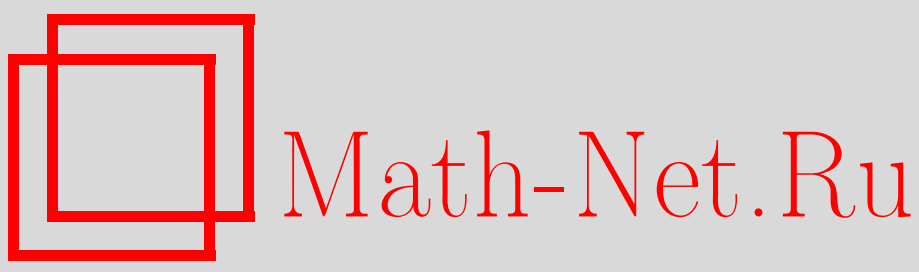

В. В. Голышев, Вариации Римана-Роха, Изв. РАН. Сер. матем., 2001, том 65, выпуск 5, 3-32

DOI: https://doi.org/10.4213/im355

Использование Общероссийского математического портала Math-Net.Ru подразумевает, что вы прочитали и согласны с пользовательским соглашением

http://www . mathnet.ru/rus/agreement

Параметры загрузки:

IP : 54.224 .135 .184

26 апреля 2023 г., 08:47:05 
УДК $512.725 .4+512.732 .6$

\title{
В. В. Голышев
}

\section{Вариации Римана-Роха}

\begin{abstract}
Построено зеркальное соответствие, сопоставляющее парам вида $(V, C)$, где $V$ - многообразие и $C$ - комплекс плотно фильтрованных расслоений на нем, вариации, т.е. локальные системы, $D$-модули или $l$-адические пучки. Рассматриваются многообразия Калаби-Яо - полные пересечения гиперповерхностей в проективном пространстве. В частном случае комплекса, квазиизоморфного касательному расслоению на общем полном пересечении типа Калаби-Яо, такая конструкция дает вариацию относительных когомологий в зеркально двойственном пучке, называемую в настоящей работе вариацией Римана-Роха. Локальная система Римана-Роха некоммутативно кодирует сведения об эйлеровых характеристиках всех пучков вида $\mathscr{O}(i)$, т.е. отражает содержание теоремы Римана-Роха для дивизориальной подрешетки в $K$-группе.
\end{abstract}

Библиография: 34 наименования.

\section{Введение}

0.1. Два формализма. Любая теория зеркальной симметрии (при том, что она исходит из своей частной эмпирики и преследует свои специфические цели) так или иначе соотносит два взгляда на когомологии алгебраического многообразия, зависяшего от параметра, две группы алгебро-геометрических теорий.

Одна теория - конструктивная. Это теория вырождения многообразия, варьируюшегося по параметру. Ее проявления в конкретных теориях когомологий-теория вырождения структур Ходжа, теория локальной монодромии в $l$-адических пучках. Если рассматривать вариации по одномерному параметру, то в каждой из этих теорий на когомологиях возникает действие алгебры $\mathrm{sl}_{2}$ (в комплексном случае она "горизонтально" действует на ромбе Ходжа).

Другая теория - когерентная (с точки зрения ромба Ходжа - "вертикальная"). Согласно сильной теореме Лефшеца с (очень обильным) классом дивизоров связано действие алгебры $\mathrm{sl}_{2}$ на когомологиях многообразия.

Центральным феноменом зеркальной симметрии можно считать именно это сходство двух $\mathrm{sl}_{2}$-формализмов (отмеченное давно [14], но до сих пор не проясненное).

Частью чего являются эти $\mathrm{sl}_{2}$-формализмы в каждой из двух теорий? В конструктивной теории, если рассматривать вариации с многомерным параметром, каждому направлению подхода к точке самого глубокого вырождения соответствует своя алгебра $\mathrm{sl}_{2}$. Эти $\mathrm{sl}_{2}$ организуются в редуктивную алгебру Ли, связанную с группой глобальной монодромии вариации.

Ответ на вопрос, можно ли расширить теорему Лефшеца в когерентной теории до бо́льших алгебр Ли или до групп Ли, в принщипе положительный (см. [26], [13]):

Работа выполнена при частичной поддержке Американского фонда гражданских исследований и развития для государств - членов СНГ (грант № RM1-2089).

(C) в.в. Голышев, 2001 
в когерентной теории имеются зеркальные двойники алгебр Ли и групп Ли из конструктивной теории. Однако эти алгебры Ли и группы Ли вторичны: первичный объект, из которого они извлекаются, - сама вариация. Главный вопрос зеркальной симметрии - как правильно построить по когерентным данным на многообразии абстрактную вариацию?

0.2. Семейство многообразий или абстрактная вариация. Обратимся к исторически первому примеру - квинтик в $\mathbb{P}^{4}$ (см., например, [12]). Наиболее распространенная постановка вопроса такова: какая конструкция сопоставляет семейству всех квинтик в $\mathbb{P}^{4}$ однопараметрическое семейство вырожденных многообразий Калаби-Яо, задаваемых уравнением

$$
\sum_{i=0}^{4} x_{i}^{5}+5 \psi \prod_{i=0}^{4} x_{i}=0 ?
$$

Можно сформулировать задачу иначе: указать конструкщию, сопоставляющую комплексам векторных расслоений на квинтике $Q$ вариации так, что комплекс

$$
0 \rightarrow \text { касательное расслоение к }\left.\mathbb{P}^{4}\right|_{Q} \rightarrow \text { нормальное расслоение к } Q \text { в } \mathbb{P}^{4} \rightarrow 0
$$

переходит в мотивную вариачию, реализующуюся в средних когомологиях слоя семейства (0.2.1).

С точки зрения когерентной теории мы расширили постановку вопроса: аргумент зеркального преобразования раньше сводился к инварианту многообразия, а теперь пробегает целый класс объектов. Однако с точки зрения конструктивной теории мы проиграли в конкретности: построить абстрактную вариацию и доказать, что она мотивная, т.е. может быть реализована в каком-либо геометрическом семействе, еще не значит избрать из семейств, в которых она может быть реализована, единственное на роль зеркального партнера для исходного когерентного комплекса.

0.3. Требования к конструкции. Желательно, чтобы искомая конструкция:

а) не выходила "за пределы алгебраической геометрии", т.е. не привлекала без острой необходимости аналитической, дифференциальной, симплектической и др. геометрий;

б) не использовала понятий, введенных в обиход самой теорией зеркальной симметрии (квантовые когомологии, фробениусовы многообразия и пр.);

в) для частного случая комплекса, подобного комплексу (0.2.1), давала вариации, предсказанные другими картинами зеркальной симметрии.

Основная цель настоящей работы - указать в случае полных пересечений такую конструкцию зеркальных вариаций (см. п. 1.4) и продемонстрировать, как она согласуется с другими известными построениями: с гипотетическим категорныс.м соответствием, предсказывающим свойства монодромии в зеркальных семействах (см. [25]); с исчислением рациональных кривых (это подход исчислительной алгебраической геометрии, и одновременно теоретико-полевой подход); с торической зеркальной симметрией, явно (в терминах линейно-геометрической двойственности) строящей по одному семейству другое. 
0.4. Обзор результатов. В $\S 1$ с алгебраическим многообразием Калаби-Яо (полным пересечением гиперповерхностей в проективном пространстве) связывается некоторая вариация - локальная система Римана-Роха. Приведенные локальные системы Римана-Роха являются неприводимыми, жесткими и квазиунипотентными (п. 1.4.3). Н. Катц [23] изучил такие системы; он доказал, что они (следовательно, и их подъемы) геометричны, т.е. реализуются в послойных когомологиях некоторого пучка алгебраических многообразий.

Работа с локальными системами Римана-Роха, введенными в $\S 1$, была бы затруднительна, так как локальные системы не образуют теорию с шестью операциями. В $\S 2$ введены антиканонические $D$-модули Римана-Роха многообразий $\Phi а-$ но. Они гипергеометрические и согласно философии Катца-Лозера-Сабба-Габбера следуют по простоте за модулями эйлеровой характеристики 0. Их монодромии дают антиканонические локальные системы Римана-Роха. Следовательно, антиканонический $D$-модуль Римана-Роха многообразия Фано геометричен, т.е. происходит из уравнения Пикара-Фукса. Рассмотрим теперь $D$-модуль, считаюший рациональные кривые (эта дивизориальная вытяжка из квантовых когомологий сообшена Д. О. Орловым). Установим соответствие между одним $D$-модулем и другим. В простейшем случае считают только прямые (в п. 2.3 мы приводим стандартное вычисление с грассманианами; см. [2]), и ответ дается в теореме 2.2.4. (Справедливость обшего утверждения должна вытекать из результатов Гивенталя [11].) Затем вводим собственно $D$-модули Римана-Роха многообразий Калаби-Яо и Фано. Связь считаюшего модуля и модуля Римана-Роха для многообразий Фано описывается теоремой 2.4.3. Монодромия $D$-модуля Римана-Роха многообразия Калаби-Яо дает локальную систему Римана-Роха этого многообразия. В случае же многообразия Фано $D$-модуль Римана-Роха иррегулярен, не сводится к своей монодромии (что и служит главной причиной перехода от локальных систем к $D$-модулям) и не геометричен. Многообразиям Фано сопоставляются дикие вариаичи.

В $\S 3$ введены "пучки Римана-Роха" - гипергеометрические превратные $l$-адические пучки на торе, являющиеся аналогами $D$-модулей Римана-Роха. Согласно основному результату Катца из [23, гл. 8] такие пучки геометричны, т.е. реализуются как композиционные факторы прямого образа постоянного пучка в некотором однопараметрическом семействе многообразий. Торическая зеркальная симметрия (см. [1], [3], [4]) связывает с полными пересечениями в проективных пространствах однопараметрическое семейство полных пересечений в торическом многообразии, двойственном к проективному пространству. Необходимо установить связь пучка Римана-Роха с прямым образом постоянного пучка в этом однопараметрическом семействе. Теорема 3.5.1 устанавливает такую связь на уровне следовых функций в случае одной гиперповерхности, а теорема 3.5 .5 - в обшем случае.

0.5. Как следует обобщать конструкцию из п. 1.4. Для унификации рассуждений удобно использовать язык $E$-объектов Катца [20]. (Теория $E$-объектов сушествует лишь гипотетически, но это не мешает ей кодировать однотипные законные действия в $D$-модульной и $l$-адической теориях. Объект-экспонента $E$ реализуется в первой теории как $D$-модуль $\mathscr{D} / \mathscr{D}\left(\frac{\partial}{\partial z}-1\right)$, во второй - как пучок Артина-Шрайера на $\mathbf{G}_{\mathbf{a}} \cdot$ )

Последовательно определим операцию $\mathscr{M}$ на расширяющихся классах объектов. 
1. Линейнье расслоения. Пусть $T=\operatorname{Hom}\left(\mathrm{NS}^{\vee}(X), \mathbf{G}_{\mathbf{m}}\right)$ - тор, двойственный к тору Нерона-Севери многообразия $X$. Класс линейного расслоения $L$ определяет элемент $\mathrm{NS}(X)$, т.е. функционал на $\mathrm{NS}^{\vee}(X)$, и, следовательно, морфизм

$$
\kappa(L): \mathbf{G}_{\mathbf{m}} \rightarrow T
$$

Пучковая зеркальная симметрия $\mathscr{M}$ сопоставляет расслоению $L$ подходящий прямой образ ограничения объекта-экспоненты $E$ на $\mathbf{G}_{\mathbf{m}}$ относительно морфизма $\kappa$ :

$$
\mathscr{M}(L)=\kappa(L)_{?}\left(\left.E\right|_{\mathbf{G}_{\mathbf{m}}}\right) .
$$

(В примере с полными пересечениями пучку $\mathscr{O}(1)$ соответствует ограничение объекта-экспоненты на $\mathbf{G}_{\mathbf{m}}$, пучку $\mathscr{O}\left(d_{j}\right)$ соответствует его прямой образ при $d_{j}$-листном куммеровом накрытии и т.д.)

Введем “дуальную” операцию $\mathscr{M}^{\vee}$; для этого двойственный объект-экспоненту $E^{\vee}$ будем отображать подходящим прямым образом относительно морфизма $\kappa^{\vee}(L) \stackrel{\text { def }}{=} \kappa\left(L^{\vee}\right)$ :

$$
\mathscr{M}^{\vee}(L)=\kappa^{\vee}(L)_{?}\left(E^{\vee} \mid \mathbf{G}_{\mathbf{m}}\right) .
$$

2. Фильтрованные векторные расслоения. Структура флага на векторном расслоении $F$ на $X$ определяет набор линейных расслоений $F_{i}$ (присоединенные факторы), а каждое из них - некий $E$-объект $E_{i}$. Расслоению $F$ будем сопоставлять свертку всех $E_{i}$ на $T$.

3. Комплекс фильтрованных векторных расслоений. Пусть

$$
F^{\bullet}=\cdots \rightarrow F^{2 j} \rightarrow F^{2 j+1} \rightarrow \cdots .
$$

Комплексу $F^{\bullet}$ сопоставим объект

$$
\mathscr{M}\left(F^{\bullet}\right)=*_{j} \mathscr{M}\left(F^{2 j}\right) * *_{j} \mathscr{M}^{\vee}\left(F^{2 j+1}\right) .
$$

В $\S 3$ эта конструкция применяется, с необходимыми уточнениями, в $l$-адической реализации к комплексу

$$
0 \rightarrow \mathscr{O} \rightarrow \oplus \mathscr{O}(1) \rightarrow \bigoplus_{j} \mathscr{O}\left(d_{j}\right) \rightarrow 0
$$

фильтрованных векторных расслоений на многообразии Калаби-Яо $X$ - полном пересечении гиперповерхностей степеней $d_{j}$ в $\mathbb{P}^{d}$. Результат назван там скрученнымм неприведенным пучком Римана-Роха.

В настоящей работе не даются окончательные ответы на многие вопросы, например: как уточнить нашу конструкцию? каким прямым образом лучше пользоваться (или $\kappa_{!}$, или $\kappa_{*}$ )? какой сверткой пользоваться? по какой подкатегории факторизовать конструктивную категорию на $T$, чтобы получить правильную область значений для зеркальной симметрии $\mathscr{M}$ ? как сделать дальнейшие шаги и определить (используя принцип расщепления) $\mathscr{M}$ на расслоениях без структуры фильтрачий, на комплексах расслоений и т.д.? 
0.6. Замечания. Идея конструирования геометрических вариаций из элементарных строительных блоков - вариаций Куммера и вариаций Артина-Шрайера восходит к работам Вейля-Ленга. Это направление было систематически разработано Катцем (см. [20], [22]).

Мысль, что геометрическую вариацию зеркальной симметрии можно строить формально как преобразование Меллина от когерентных инвариантов многообразия, принадлежит, по-видимому, Аспинуоллу, Грину и Моррисону.

Задача систематического поиска и исследования семейств многообразий, исчезающие циклы в которых соответствовали бы элементам исключительных наборов, была поставлена уже давно. М. Концевич заметил, что именно такое соответствие и реализует зеркальная симметрия.

Автор приносит благодарность А. Бондалу, А. Городенщеву, В. Лунщу и Д. Орлову за ценные замечания и объяснения, особая благодарность - В. Острику, который внимательно прочел рукопись работы и сделал много критических замечаний и предложений, и М. Цфасману за постоянную поддержку. Автор выражает также благодарность Независимому Московскому университету за поддержку семинара "Представления Галуа - зеркальная симметрия".

\section{§ 1. Локальные системы}

1.1. Катцевы локальные системы. В настоящей рабте рассмотрены многообразия, определенные над полем $\mathbb{C}$.

Пусть $G$ - группа.

ОПРЕДЕЛЕНИЕ 1.1.1. Назовем представление $\phi: G \rightarrow \mathrm{GL}(V)$ жестким относительно совокупности $\left\{g_{0}, \ldots, g_{n}\right\} \subset G$, если для всякого представления $\phi^{\prime}: G \rightarrow \mathrm{GL}(V)$ такого, что

$$
\forall i \quad \phi\left(g_{i}\right) \text { сопряжен } \phi^{\prime}\left(g_{i}\right),
$$

имеем

$$
\phi \text { сопряжено } \phi^{\prime} \text {. }
$$

ОПРЕДЕЛЕНИЕ 1.1.2. Назовем представление $\phi: G \rightarrow \mathrm{GL}(V)$ локально жестким относительно совокупности $\left\{g_{0}, \ldots, g_{n}\right\} \subset G$, если для всякого достаточно близкого к $\phi$ представления $\phi^{\prime}: G \rightarrow \mathrm{GL}(V)$ такого, что

$$
\forall i \quad \phi\left(g_{i}\right) \text { сопряжен } \phi^{\prime}\left(g_{i}\right),
$$

имеем

$$
\phi \text { сопряжено } \phi^{\prime} \text {. }
$$

Пусть $U$ - непустое открытое подмножество полной кривой $C$ над $\mathbb{C}$.

ОПРЕДЕЛЕНИЕ 1.1.3. Назовем локальную систему $\mathscr{F}$ на $U^{\text {an }}$ локально жесткой (соответственно, жесткой), если представление монодромии

$$
\pi_{1}\left(U^{\mathrm{an}}\right) \rightarrow \mathrm{GL}(\text { слой } \mathscr{F})
$$

локально жестко (соответственно, жестко) относительно множества обходов вокруг точек из $C^{\text {an }} \backslash U^{\text {an }}$. 
Tеорема 1.1.4 [23, п. 1.1.2]. Пусть $S$ - непустое конечное подмножество $\mathbb{P}^{1}(\mathbb{C}), \quad U$ - соответствующее открытое дополнение, $j$ - открытое вложение. Пусть $\mathscr{F}$ - неприводимая локальная система на $U^{\text {an }}$ ранга не меньше 1.

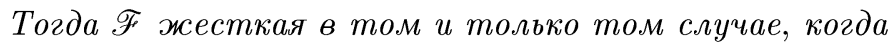

$$
\chi\left(\left(\mathbb{P}^{1}\right)^{\text {an }}, j_{*}(\mathscr{E} n d(\mathscr{F}))\right)=2 .
$$

ЗАмЕчАниЕ 1.1.5. Как видно из доказательства этой теоремы, требование жесткости в ее формулировке можно ослабить до требования локальной жесткости. В частности, верно

СлЕДСТВИЕ 1.1.6. Нетривиальная неприводимая локальная система на непустом аффинном подмножестве $\mathbb{P}^{1}$ жестка тогда и только тогда, когда она локально жестка.

ОПРЕДЕЛЕНИЕ 1.1.7. Неприводимая локальная система $\mathscr{L}$ на $U$ называется катцевой, если она жесткая и все ее локальные монодромии (т.е. образы обходов вокруг точек из $C^{\text {an }} \backslash U^{\text {an }}$ в представлении монодромии) квазиунипотентны.

ОПРЕДЕЛЕНИЕ 1.1.8. Локальная система $\mathscr{L}_{0}$ на $U_{0}$ называется квазикатцевой, если найдутся катцева локальная система $\mathscr{L}$ на аффинной кривой $U$ и неразветвленное накрытие $\pi: U_{0} \rightarrow U$ такие, что $\mathscr{L}_{0} \cong \pi^{*}(\mathscr{L})$. (Подъем локальной системы, разумеется, соответствует ограничению представления монодромии на подгрупшу $\pi_{1}\left(U_{0}\right)$ в $\pi_{1}(U)$.)

Используя формулу Римана-Роха, свяжем с многообразиями Калаби-Яо, являющимися полными пересечениями в проективных пространствах, катцевы локальные системы.

1.2. Формула $H$-рядов. Пусть $V$ - линейное пространство над полем $k$, $\operatorname{dim} V=n+1$. Пусть в пространстве $V$ действуют два оператора $T$ и $S$ такие, что:

i) $\operatorname{rk}(1-S)=1$, т.е. $S$ - псевдоотражение;

ii) $\sum T^{i}(1-S)(V)=V$.

Иначе говоря, если вектор $v$ - образуюшая одномерного пространства $(1-S) V$, то требуется, чтобы $v$ был циклическим вектором для $T$.

Определим ряд $H_{T, S}$ следующим образом:

$$
H_{T, S}(t)=1+\sum_{i=1}^{\infty} \frac{(1-S) T^{i} v}{v} t^{i}=1+\sum_{i=1}^{\infty} h_{i} t^{i} \in k[[t]] .
$$

ТЕОРема 1.2.1 (формула $H$-рядов). Выполнено равенство рядов

$$
H_{T, S}(t) \operatorname{det}(1-t T)=\operatorname{det}(1-t S T) .
$$


ДокАЗАТЕЛЬСТво. Имеем

$$
\begin{aligned}
H_{T, S}(t) & =\frac{\left(1+(1-S) t T(1-t T)^{-1}\right) v}{v} \\
& =\frac{(1-t T+t T-t S T)(1-t T)^{-1} v}{v}=\frac{(1-t S T)(1-t T)^{-1} v}{v} .
\end{aligned}
$$

Оператор $(1-t S T)(1-t T)^{-1}$ есть сумма тождественного оператора и оператора ранга 1 , образом которого является прямая, порожденная вектором $v$. Следовательно, его детерминант равен его собственному числу, соответствуюшему $v$. Значит,

$$
\left((1-t S T)(1-t T)^{-1} v\right) / v=\operatorname{det}\left((1-t S T)(1-t T)^{-1}\right) .
$$

СлЕДСТВИЕ 1.2.2. Пусть $V$ - линейное пространство размерности $n+1$ над полем, снабженное невырожденной симметрической (или косой) формой $($; ), $T$ - невырохсденный оператор, действующий в $V$. Пусть $v$ - циклический вектор для оператора $T$ и $S$ - отражение относительно $v$ :

$$
S: x \mapsto x-(x, v) v .
$$

Тогда

$$
1+\sum_{i=1}^{\infty}\left(T^{i} v ; v\right) t^{i}=\frac{\operatorname{det}(1-t T S)}{\operatorname{det}(1-t T)} .
$$

ПРЕДЛОЖЕНИЕ 1.2.3. Пусть заданы два произвольных многочлена $P(t)$, $Q(t)$ степени $n+1$ со свободными членами, равными 1 . Пусть $W$ - линейное пространство размерности $n+1$. Существует единственная с точностью до сопряжения пара операторов $T, S \in \mathrm{GL}(W, k)$ такая, что:

i) $\operatorname{det}(1-t T)=P(t)$;

ii) $\operatorname{det}(1-t S T)=Q(t)$;

iii) $S$ - псевдоотражение;

iv) $\sum T^{i} \operatorname{Im}(1-S)=W$, т.е. прямая $\operatorname{Im}(1-S)$ является ииклической для onepamopa $T$.

ДокАЗАТЕЛьСтво. Теорема 1.2.1 показьвает, что в базисе $\left\{T^{i}\left(v_{0}\right) \mid i=\right.$ $0, \ldots, n\}$, где $v_{0}$ - нетривиальньй вектор на прямой $\operatorname{Im}(1-S)$, операторы $T$ и $S$ (точнее, их матрицы)

$$
\begin{aligned}
& T=\left(\begin{array}{ccccc}
0 & 0 & 0 & \ldots & -v_{n+1} \\
1 & 0 & 0 & \ldots & -v_{n} \\
0 & 1 & 0 & \ldots & -v_{n-1} \\
\ldots & \ldots & \ldots & \ldots & \ldots \\
0 & 0 & 0 & \ldots & -v_{1}
\end{array}\right), \quad S=\left(\begin{array}{ccccc}
1-h_{0} & -h_{1} & -h_{2} & \ldots & -h_{n} \\
0 & 1 & 0 & \ldots & 0 \\
0 & 0 & 1 & \ldots & 0 \\
\ldots \ldots & \ldots & \ldots & \ldots & \ldots \\
0 & 0 & 0 & \ldots & 1
\end{array}\right)
\end{aligned}
$$

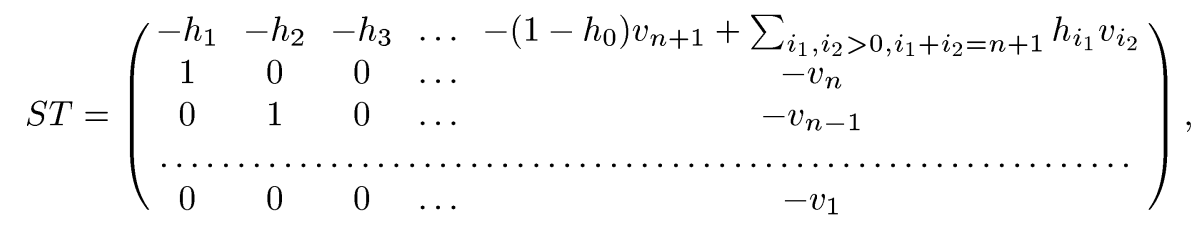

определяются однозначно по $P(t)$ и $Q(t)$. 
СлЕДСТВИЕ 1.2.4. Пусть $F_{2}=\langle s, t\rangle-$ свободная группа от двух образующих. Рассмотрим представление $\phi: F_{2} \rightarrow \mathrm{GL}(W)$, определяемое тем, что $\phi(s)=S, \phi(t)=T$. Тогда ф локально жсестко относительно $\{s, t, s t\}$.

ПРЕДЛОЖЕНИЕ 1.2.5. Пусть многочлены $P$ и $Q$ взаимно просты. Тогда $W$ неприводимо как $F_{2}$-представление.

ДокАЗАТЕльСтво. Пусть $W_{0}$ - нетривиальное подпредставление $W$. Тогда образуюшая $s$ индуцирует на $W_{0}$ тождественный оператор, иначе $(1-S) W_{0} \neq 0$ и $\sum T^{i}(1-S) W_{0}=W_{0}=W$. Значит, ограничения $S$ и $S T$ на $W_{0}$ совпадают, следовательно, операторы $S$ и $S T$ имеют обшее собственное число.

1.3. Полные пересечения в проективных пространствах. Пусть $V$ гладкое многообразие в $\mathbb{P}^{r}$, являюшееся полным пересечением гиперповерхностей степеней $d_{j}$. Мы будем предполагать, что его размерность больше 2.

Числа Ходжа $H^{p, q}$ многообразия $V$ могут отличаться от чисел Ходжа проективного пространства той же размерности лишь на горизонтали $p+q=\operatorname{dim} V$. Действительно, по теореме Лефшеца когомологии при каждом сечении гиперповерхностью могут меняться только в средней размерности.

Имеем $\operatorname{Pic}(V)=\mathbb{Z}$ (теорема Лефшеца), и канонический класс $V$ по формуле присоединения равен $\mathscr{O}\left(-r+\sum d_{j}-1\right)$. Таким образом, $V$ - многообразие Фано тогда и только тогда, когда $\sum d_{j} \leqslant r$.

ПреДЛОЖЕНИЕ 1.3.1. Пусть $V$ - многообразие Фано. Имеет место формула для производящего ряда для значений многочлена Гильберта в неотрицательных цельх точках:

$$
\sum_{i=0}^{\infty} \chi(\mathscr{O}(i)) t^{i}=(1-t)^{-r-1} \prod_{j}\left(1-t^{d_{j}}\right)
$$

ДокаЗАтЕльСтво. Будем использовать формулу Римана-Роха-Хирцебруха. Род Тодда представляется как формальный ряд от образующей Пикара:

$$
\operatorname{td}(\mathscr{T})=\frac{g^{\operatorname{dim} V+1}}{\prod d_{j}} \frac{\prod\left(1-e^{-d_{j} g}\right)}{\left(1-e^{-g}\right)^{r+1}}
$$

где $g=c_{1}(\mathscr{O}(1))$.

Производящий (по $t)$ ряд, который вычисляется, - это $\operatorname{dim}(V)$-й коэффициент ряда

$$
\frac{1}{1-t e^{g}} g^{\operatorname{dim} V+1} \prod\left(1-e^{-d_{j} g}\right) /\left(1-e^{-g}\right)^{r+1},
$$

или, что важнее, это вычет

$$
\operatorname{Res}_{g=0} \frac{1}{1-t e^{g}} \frac{\prod\left(1-e^{-d_{j} g}\right)}{\left(1-e^{-g}\right)^{r+1}} d g .
$$

После экспоненциальной замены переменной $z=e^{-g}$ имеем

$$
\sum_{i=0}^{\infty} \chi(\mathscr{O}(i)) t^{i}=\operatorname{Res}_{z=1}\left(-\frac{1}{1-t / z} \frac{\prod\left(1-z^{d_{j}}\right)}{(1-z)^{r+1}} \frac{1}{z} d z\right)=\operatorname{Res}_{z=1}\left(-\frac{1}{z-t} \frac{\prod\left(1-z^{d_{j}}\right)}{(1-z)^{r+1}} d z\right) .
$$


Заметим, что при фиксированном $t$ выражение, от которого берется вычет, не

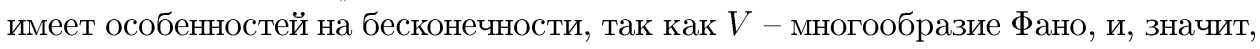
выполняется $\sum d_{j}<r+1$. Следовательно, вычет в 1 равен вычету в $t$ с противоположным знаком, и окончательно имеем

$$
\sum_{i=0}^{\infty} \chi(\mathscr{O}(i)) t^{i}=\frac{\prod\left(1-t^{d_{j}}\right)}{(1-t)^{r+1}}
$$

Если же $V$ - многообразие Калаби-Яо, то, как легко проверить, у правой части в бесконечности появляется вычет, равный 1 или -1 . Значит, верно

ПРЕДЛОЖЕНИЕ 1.3.2. Для производящего ряда по значениям в иельх точках многочлена Гильберта многообразия Калаби-Яо - полного пересечения гиперповерхностей степеней $d_{j}$ в $\mathbb{P}^{r}$ - вьполняется

$$
1+\sum_{i=1}^{\infty} \chi(\mathscr{O}(i)) t^{i}=\frac{\prod\left(1-t^{d_{j}}\right)}{(1-t)^{r+1}}
$$

1.4. Построение локальных систем по расслоениям. Пусть $V$ - многообразие такое, что $\operatorname{Pic} V=\mathbb{Z}$. Через $\mathscr{O}(1)=\mathscr{O}(1)_{V}$ обозначим положительную образующую в группе Пикара, через $\mathscr{O}(i)$ - ее $i$-ю тензорную степень. Это обозначение не приведет к путанище, так как речь всегда будет идти о пучках на $V$, а не на объемлющем проективном пространстве.

ПРЕДЛОЖЕНИЕ 1.4.1. Размерность подпространства в $K_{0}(V) \otimes \mathbb{Q}$, порожденного пучками $\mathscr{O}(i)$, равна $\operatorname{dim}(V)+1$.

ДоКАЗАтЕльство. Действительно, $\operatorname{ch} \mathscr{O}(i)=e^{i g}$. Из рассмотрения определителя Вандермонда следует, что это пространство имеет размерность не меньше $\operatorname{dim}(V)+1$, но и не больше $\operatorname{dim}(V)+1$, так как оно порождается степенями $g$.

Пусть $C^{\bullet}$ - комплекс расслоений на $V$ :

$$
C^{\bullet}=\cdots \rightarrow C^{i} \rightarrow C^{i+1} \rightarrow \cdots
$$

такой, что для всякого $i$ расслоение $C^{i}$ - это последовательное расширение полуобильных линейных расслоений. Любому такому комплексу можно сопоставить элемент $а$ свободной абелевой группы $\mathscr{A}$, порожденной обильными линейными расслоениями $\mathscr{O}(1), \mathscr{O}(2), \ldots$; таким образом сложим все обильные линейные расслоения, входящие композиционными факторами в $\oplus C^{\text {чет }}$, и вычтем все обильные линейные расслоения, входящие композищионными факторами в $\oplus C^{\text {нечет }}$.

Положим

$$
a=\sum_{i} a_{i}^{+} \mathscr{O}(i)-\sum_{i} a_{i}^{-} \mathscr{O}(i), \quad a_{i}^{+} \geqslant 0, \quad a_{i}^{-} \geqslant 0 .
$$

Пусть первый класс Чженя этого комплекса равен нулю, иначе говоря, выполняется условие

$$
\sum_{i} i\left(a_{i}^{+}-a_{i}^{-}\right)=0
$$


Положим

$$
\begin{aligned}
& X_{0}^{+}(t)=\prod_{i>0}\left(1-t^{i}\right)^{a_{i}^{+}}, \\
& X_{0}^{-}(t)=\prod_{i>0}\left(1-t^{i}\right)^{a_{i}^{-}} .
\end{aligned}
$$

Избавимся от обших делителей $X_{0}^{+}(t)$ и $X_{0}^{-}(t)$. Положим

$$
\begin{aligned}
& X^{+}(t) \stackrel{\text { def }}{=} X_{0}^{+}(t) / \text { НОД }\left(X_{0}^{+}(t), X_{0}^{-}(t)\right), \\
& X^{-}(t) \stackrel{\text { def }}{=} X_{0}^{-}(t) / \text { НОД }\left(X_{0}^{+}(t), X_{0}^{-}(t)\right),
\end{aligned}
$$

где НОД однозначно определяется так, чтобы его свободный член был равен 1.

Пусть $W$ - пространство размерности

$$
\begin{aligned}
\operatorname{dim} W & =\operatorname{deg} X^{+}(t)-\operatorname{deg} \text { НОД }\left(X_{0}^{+}(t), X_{0}^{-}(t)\right) \\
& =\operatorname{deg} X^{-}(t)-\operatorname{deg} \text { НОД }\left(X_{0}^{+}(t), X_{0}^{-}(t)\right) .
\end{aligned}
$$

ПРЕДЛОЖЕНИЕ 1.4.2. Существует единственная с точностью до одновременного сопряжения пара операторов $\mathbf{X}^{-}, \mathbf{X}^{+} \in \mathrm{GL}(W)$ mаких, что:

i) $\operatorname{det}\left(1-t \mathbf{X}^{-}\right)=X^{-}(t)$;

ii) $\operatorname{det}\left(1-t \mathbf{X}^{+}\right)=X^{+}(t)$;

iii) $\operatorname{rk}(1-\mathbf{X})=1$, где $\mathbf{X}=\mathbf{X}^{+}\left(\mathbf{X}^{-}\right)^{-1}$;

iv) $\sum_{j}\left(\mathbf{X}^{-}\right)^{j}(\operatorname{Im}(1-\mathbf{X}))=W$.

ДоказАТЕЛьство. Заменив обозначения $\mathbf{X}_{0}^{-}$на $T$ и $\mathbf{X}_{0}^{+}$на $S T$, приходим к условиям п. 1.2. Теперь воспользуемся предложением 1.2.3.

Пусть $U=\mathbb{P}^{1} \backslash\{0,1, \infty\}$. Будем отождествлять локальные системы на $U$ с представлениями свободной группы $F_{2}=\left\langle s_{0}, s_{\infty}\right\rangle$. Рассмотрим представление $\rho: F_{2} \rightarrow \mathrm{GL}(W)$ такое, что

$$
\rho\left(s_{\infty}\right)=\mathbf{X}^{-}, \quad \rho\left(s_{1}\right)=\mathbf{X}, \quad \rho\left(s_{0}\right)=\left(\mathbf{X}^{+}\right)^{-1} .
$$

Таким образом, по комплексу расслоений специального вида построена локальная система. Рассмотрим один частный случай этой конструкции.

Пусть, как и в п. 1.3, $V$ - многообразие Калаби-Яо - полное пересечение гиперповерхностей степеней $d_{j}$ в $\mathbb{P}^{r}$. Такому многообразию можно сопоставить следующий комплекс вида (1.4.1) (квазиизоморфньй касательному расслоению на $V$ ):

$$
0 \rightarrow \mathscr{O} \rightarrow \mathscr{O}(1) \oplus \mathscr{O}(1) \oplus \cdots \oplus \mathscr{O}(1) \rightarrow \mathscr{O}\left(d_{j}\right) \rightarrow 0
$$

для которого имеем

$$
a=-(d+1) \mathscr{O}(1)+\sum_{j} \mathscr{O}\left(d_{j}\right)
$$

Такая конструкция сопоставит комплексу (1.4.2) локальную систему; назовем ее локальной системой Римана-Роха многообразия $V$ и обозначим $\mathscr{L}_{R R}(V)$. 
Теорема 1.4.3. Локальные системы Римана-Роха $\mathscr{L}_{R R}(V)$ неприводимы, жестки и квазиунипотентны.

ДокАЗАТЕЛЬСТвО. Неприводимость следует из предложения 1.2.5. Жесткость следует из следствий 1.1.6, 1.2.4 и неприводимости. Квазиунипотентность видна из определения.

ОПРЕДЕЛЕНИЕ 1.4.4. Пусть $M$ - многообразие Фано. Антиканонической локальной системой Римана-Роха $\mathscr{A} \mathscr{L}_{R R}(M)$ называется локальная система Римана-Роха общего сечения $V$ антиканонического класса на $M$ :

$$
\mathscr{A} \mathscr{L}_{R R}(M) \stackrel{\text { def }}{=} \mathscr{L}_{R R}(V) \text {. }
$$

1.5. Подкрутка и отражение. Пусть $V$-многообразие Калаби-Яо-полное пересечение гиперповерхностей степеней $d_{j}$ в $\mathbb{P}^{r}$. По-прежнему пишем $\mathscr{O}(i)$ вместо $\mathscr{O}(i)_{V}$.

Пусть $W_{\mathscr{O}}-$ подпространство в $K_{0}(V) \otimes \mathbb{Q}$, порожденное классами пучков $\mathscr{O}(i)$. Согласно предложению 1.4.1 его размерность равна $\operatorname{dim}(V)+1$. Эйлерова характеристика $([A],[B])=\sum(-1)^{i} \operatorname{Hom}^{i}(A, B)$ пары когерентных пучков $A$ и $B$ на $V$ задает билинейную форму $($,$) на W_{\mathscr{O}}$; назовем ее формой Римана-Роха. (Квадратными скобками здесь обозначен класс объекта в $K_{0}(X) \otimes \mathbb{Q}$.)

ПреДЛОЖЕнИЕ 1.5.1. Форма Римана-Роха является в зависимости от размерности $V$ либо симметричной (в четномерном случае), либо косой (в нечетномерном случае).

ДокАЗАТЕЛЬСтво. Это легко следует из двойственности Серра.

На пространстве $W_{\mathscr{O}}$ естественно действуют операторы $T$ и $S$. Первьй оператор, $T$, - это оператор умножения на класс $\mathscr{O}(1)$ (назовем его подкруткой),

$$
T:[\mathscr{F}] \mapsto[\mathscr{F} \otimes \mathscr{O}(1)]
$$

здесь [ ] обозначает класс пучка. Второй оператор, $S$, - это отражение

$$
x \mapsto x-(x,[\mathscr{O}])[\mathscr{O}]
$$

относительно $[\mathscr{O}]=1$.

Существует единственная с точностью до изоморфизма локальная система $\mathscr{L}\left(S, T, W_{\mathscr{O}}\right)$ на $\mathbb{P}^{1} \backslash\{0,1, \infty\}$ такая, что ее слой равен $W_{\mathscr{O}}$, а монодромии обхода вокруг $\infty$ и 1 действуют операторами $T$ и $S$ соответственно.

ПРЕДЛОЖЕНИЕ 1.5.2. Локальная система $\mathscr{L}\left(S, T, W_{\mathscr{O}}\right)$ изоморфна локальной системе Римана-Роха:

$$
\mathscr{L}\left(S, T, W_{\mathscr{O}}\right) \cong \mathscr{L}_{R R}(V)
$$


ДоКАЗАТЕЛЬСТВО. Проверим вьполнение условий предложения 1.4.2. Условие п. іiі) выполнено потому, что $S$ - (псевдо)отражение. Условие п. і) выполнено потому, что

$$
\operatorname{det}(1-t T)=\operatorname{det}\left(1-t \mathbf{X}^{-}\right)=(1-t)^{\operatorname{dim} V+1},
$$

так как оператор подкрутки является унипотентным: фильтрация на $W$ стабильна относительно подкрутки, и ее действие на присоединенных факторах тривиально. Условие п. іv) выполнено потому, что по построению $\sum T^{i}(\operatorname{Im}(1-S))=W_{\mathscr{O}}$. Наконец, запишем операторы $T$ и $S$ в базисе, состояшем из классов пучков $\mathscr{O}(i)$. Элементы верхней строки матрицы оператора $S$ - это $h_{i}=(\mathscr{O}, \mathscr{O}(i))$. Из теоремы 1.2.1 и предложения 1.3.2 следует, что вьполняется условие п. ii).

1.6. Исключительные наборы. Пусть $X$ - алгебраическое многообразие. Обозначим через $\operatorname{coh}(X)$ категорию когерентных пучков на $X$, через $\mathscr{D}^{b}(X)$ - ограниченную производную категорию $\operatorname{coh}(X)$.

Любая производная категория $\mathscr{D}$ является триангулированной. Это значит, что заданы:

a) функтор сдвига [1]: $\mathscr{D} \rightarrow \mathscr{D}$ - аддитивная автоэквивалентность;

б) класс выделенных треугольников

$$
X \rightarrow Y \rightarrow Z \rightarrow X[1]
$$

удовлетворяющий определенным аксиомам (см. [31]).

Аддитивный функтор $F: \mathscr{D} \rightarrow \mathscr{D}^{\prime}$ из одной триангулированной категории в другую называется точныцм, если он коммутирует с функтором сдвига и переводит выделенные треугольники в выделенные.

ОПРЕДЕЛЕниЕ 1.6.1. Группа классов изоморфизма точных эквивалентностей $F: \mathscr{D}^{b}(X) \rightarrow \mathscr{D}^{b}(X)$ называется группой автоэквивалентностей категории $\mathscr{D}^{b}(X)$ и обозначается $\operatorname{Auteq}\left(\mathscr{D}^{b}(X)\right)$.

Далее, в пп. 1.6.2-1.6.6, использован материал из работы [6].

Через $\operatorname{Hom}^{i}(X, Y)$ обозначим $\operatorname{Hom}(X, Y[i])$.

ОПРеДЕЛЕниЕ 1.6.2. Объект $E \in \operatorname{Ob}\left(\mathscr{D}^{b}(X)\right)$ называется исключительныц, если он удовлетворяет условиям

$$
\operatorname{Hom}^{i}(E, E)=0 \text { при } i>0, \operatorname{Hom}^{0}(E, E)=\mathbb{C} .
$$

ОПРЕДЕЛЕНИЕ 1.6.3. Упорядоченный набор исключительных объектов $E_{0}, \ldots$ $\ldots, E_{n}$ называется исключительныцм, если для всякого $i$

$$
\operatorname{Hom}^{i}\left(E_{j}, E_{k}\right)=0 \text { при } j>k .
$$

ПРЕДЛОЖЕНИЕ 1.6.4 (см. [5], [18]). На проективных пространствах и квадриках существуют исключительные наборы.

ОПРЕДЕЛЕНИЕ 1.6.5. Исключительный набор называется полным, если он порождает всю производную категорию. 
ОПРЕДЕЛЕНИЕ 1.6.6. Мы назовем базис $v_{0}, \ldots, v_{n}$ линейного пространства $V$, снабженного билинейной формой $\chi$, полуортонормированным, если $\chi\left(v_{i}, v_{j}\right)=0$ при $i>j$ и $\chi\left(v_{k}, v_{k}\right)=1$ для всех $k$.

Классы элементов полного исключительного набора образуют полуортонормированный базис в $K_{0}(X) \otimes \mathbb{Q}$ относительно формы Римана-Роха.

ПРЕДЛОЖЕнИЕ 1.6.7. Пусть $A$ - матрица билинейной формы ұ в полуортонормированном базисе $v_{0}, \ldots, v_{n}$ пространства $V$. Пусть $\chi_{s}-$ симметризачия формы ұ, т.е.

$$
\chi_{s}\left(w_{1}, w_{2}\right)=\chi\left(w_{1}, w_{2}\right)+\chi\left(w_{2}, w_{1}\right),
$$

и пусть $I_{0}, \ldots, I_{n}$ - отражения относительно векторов $v_{0}, \ldots, v_{n}$ в ортогональном пространстве $\left(V, \chi_{s}\right)$,

$$
I_{j}: v \mapsto v-\chi_{s}\left(v, v_{j}\right) v_{j}
$$

Тогда имеем

$$
I_{0} I_{1} \ldots I_{n}=-A^{-1} A^{t}
$$

ДоКАЗАТЕЛЬСТво (устное сообшение В. Острика). Введем следуюшие обозначения:

$A_{[0, j]}$ - матрица, получаемая из $A$ пересечением первых $j+1$ строк и первых $j+1$ столбцов;

$A_{[j+1, n]}$ - матрица, получаемая из $A$ пересечением последних $n-j$ строк и последних $n-j$ столбцов;

$a_{[0, j]}$ - подстолбец $j$-го столбца матрищы $A$, состояший из элементов, расположенных строго вьше диагонали;

$a_{[j, n]}$ - подстрока $j$-ой строки матрицы $A$, состоящая из элементов, расположенных строго правее диагонали.

Будем рассматривать произведения

$$
\begin{aligned}
A I_{0} & =\left(\begin{array}{cccc}
1 & A_{01} & A_{02} & \ldots \\
0 & 1 & \ldots & \ldots \\
0 & 0 & 0 & 1
\end{array}\right)\left(\begin{array}{cccc}
-1 & -A_{01} & -A_{02} & \ldots \\
0 & 1 & 0 & \ldots \\
0 & 0 & 0 & 1
\end{array}\right) \\
& =\left(\begin{array}{cccc}
-1 & 0 & 0 & \ldots \\
0 & \ldots & \ldots & \ldots \\
0 & 0 & 0 & 1
\end{array}\right), \quad A I_{0} I_{1}, \ldots
\end{aligned}
$$

По индукции имеем

$$
\begin{aligned}
& \left(\begin{array}{ccc}
-A_{[0, j]}^{t} & 0 & 0 \\
0 & 1 & a_{[j+1, n]} \\
0 & 0 & A_{[j+2, n]}
\end{array}\right)\left(\begin{array}{crc}
1 & 0 & 0 \\
-a_{[0, j+1]}^{t} & -1 & -a_{[j+1, n]} \\
0 & 0 & 1
\end{array}\right) \\
& =\left(\begin{array}{crc}
-A_{[0, j]}^{t} & 0 & 0 \\
-a_{[0, j+1]}^{t} & -1 & 0 \\
0 & 0 & A_{[j+2, n]}
\end{array}\right)=\left(\begin{array}{ccc}
-A_{[0, j+1]}^{t} & 0 & 0 \\
0 & 1 & a_{[j+2, n]} \\
0 & 0 & A_{[j+3, n]}
\end{array}\right),
\end{aligned}
$$

и в результате получим

$$
A I_{0} I_{1} \ldots I_{n}=-A^{t} .
$$


ПРЕДЛОЖЕНИЕ 1.6.8. Пусть $A$ - матрица билинейной формы ұ в полуортонормированном базисе $v_{0}, \ldots, v_{n}$ пространства $W$. Пусть $\chi_{a}$ - кососимметризачия формы ұ, т.е.

$$
\chi_{a}\left(w_{1}, w_{2}\right)=\chi\left(w_{1}, w_{2}\right)-\chi\left(w_{2}, w_{1}\right)
$$

и пусть $I_{0}, \ldots, I_{n}$ - отражения относительно векторов $v_{0}, \ldots, v_{n}$ в симплектическом пространстве $\left(V, \chi_{a}\right)$. Тогда имеем

$$
I_{0} I_{1} \ldots I_{n}=A^{-1} A^{t}
$$

ДоКАЗАТЕЛЬСТВО полностью аналогично доказательству предложения 1.6.7.

Заметим теперь, что в случае, когда $W=K_{0}(X) \otimes \mathbb{Q}$, формула Римана-Роха дает для любой пары объектов $O_{1}, O_{2} \in \mathrm{Ob} D^{b}(X)$

$$
\chi\left(O_{1}, O_{2}\right)=\chi\left(O_{2}, O_{1} \otimes K_{X}[m]\right)
$$

Пусть $A$ - матрица формы $\chi$ в базисе из классов элементов исключительного набора. Тогда равенство

$$
x^{t} A y=y^{t} A\left(A^{-1} A^{t}\right) x
$$

показывает, что в этом же базисе матрица оператора, который задается на $K_{0} \otimes \mathbb{Q}$ функтором $\otimes K_{X}[m]$, есть $A^{-1} A^{t}$. Следовательно, справедливо

ПРЕДЛОЖЕНИЕ 1.6.9. В пространстве $K_{0} \otimes \mathbb{Q}$ :

а) в случае когда $X$ - нечетномерное проективное пространство или нечетномерная квадрика, произведение всех ортогональных отражсений относительно классов әлементов исключительного набора совпадает с умножением на $\left[K_{X}\right]$;

б) в случае когда $X$ - четномерное проективное пространство, произведение всех симплектических отражений относительно классов әлементов исключительного набора совпадает с умножением на $\left[K_{X}\right]$.

1.7. Квазикатцевы многообразия. Пусть задано нечетномерное (соответственно, четномерное) многообразие $X$, обладающее полным исключительным набором $\mathbf{E}=\left\langle E_{0}, E_{1}, \ldots, E_{n}\right\rangle$. Пусть $\chi_{\bullet}-$ симметризованная (соответственно, кососимметризованная) форма Римана-Роха. Рассмотрим линейное пространство $W=\left(K_{0}(X) \otimes \mathbb{Q}\right) / \operatorname{Ker} \chi_{\bullet}$.

Ограничение $\chi \bullet$ на $W$ также будем обозначать через $\chi_{\bullet}$.

Пусть $\mathbf{x}=\left\langle x_{0}, \ldots, x_{n}\right\rangle$ - набор различных точек на $\mathbb{A}^{1}$. Положим $U=\mathbb{A}^{1} \backslash$ $\left\{x_{0}, \ldots, x_{n}\right\}$ и рассмотрим представление

$$
\phi_{\mathbf{E}, \mathbf{x}}: \pi_{1}(U) \rightarrow \mathrm{SO}\left(W, \chi_{\bullet}\right) \quad\left(\text { соответственно, } \pi_{1}(U) \rightarrow \operatorname{Sp}\left(W, \chi_{\bullet}\right)\right)
$$

определенное тем условием, что образующая, соответствующая обходу вокруг $i$-й точки, действует отражением относительно $\left[E_{i}\right]$. Это представление определяет локальную систему $\mathscr{L}_{\mathbf{E}, \mathbf{x}}$ на $U$. 
ОПРЕДЕЛЕНИЕ 1.7.1. Назовем исключительный набор $\mathbf{E}$ квазикатиевым, если найдется такой набор точек $\mathbf{x}=\left\{x_{0}, \ldots, x_{n}\right\} \subset \mathbb{A}^{1}$, что локальная система $\mathscr{L}_{\mathbf{E}, \mathbf{x}}$ квазикатцева. Назовем многообразие $X$ квазикатцевым, если оно обладает квазикатцевым исключительным набором.

Пусть $X=\mathbb{P}^{n}, \zeta$ - первообразный корень степени $\operatorname{ind}\left(\mathbb{P}^{n}\right)=n+1$ из 1 . Рассмотрим набор $\mathbf{E}=\langle\mathscr{O}, \ldots, \mathscr{O}(n)\rangle$. Обозначим через $[n+1]$ накрытие Куммера:

$$
[n+1]: \mathbf{G}_{\mathbf{m}} \stackrel{n+1}{\longrightarrow} \mathbf{G}_{\mathbf{m}}, \quad z \mapsto z^{n+1} .
$$

ПРЕДЛОЖЕНИЕ 1.7.2. Оحраничение локальной системы $\mathscr{L}_{\mathbf{E},\left\langle 1, \zeta, \zeta^{2}, \ldots, \zeta^{n}\right\rangle}$ на $\mathbf{G}_{\mathbf{m}} \backslash\left\{1, \zeta, \zeta^{2}, \ldots, \zeta^{n}\right\}$ изоморфно $[n+1]^{*} \mathscr{A} \mathscr{L}_{R R}\left(\mathbb{P}^{n}\right)$.

ДокАЗАТЕЛЬСТво. Сперва заметим, что собственные числа локальной монодромии $\mathscr{A}_{R R}\left(\mathbb{P}^{n}\right)$ в 0 - это различные корни степени $n+1$ из 1 , следовательно, $[n+1]^{*} \mathscr{A} \mathscr{L}_{R R}\left(\mathbb{P}^{n}\right)$ продолжается в 0 , т.е. существует единственная локальная система на $\mathbb{A}^{1} \backslash\left\{1, \zeta, \ldots, \zeta^{n}\right\}$ такая, что ее ограничение на $\mathbf{G}_{\mathbf{m}} \backslash\left\{1, \zeta, \ldots, \zeta^{n}\right\}$ изоморфно $[n+1]^{*} \mathscr{A} \mathscr{L}_{R R}\left(\mathbb{P}^{n}\right)$.

Монодромия $[n+1]^{*} \mathscr{A} \mathscr{L}_{R R}\left(\mathbb{P}^{n}\right)$ задается ограничением представления $\phi_{E_{i}, x_{i}}$ на нормальную подгруппу индекса $n+1$, порождаемую оператором $T^{n+1}$ (т.е. подкруткой на антиканонический класс, или монодромией в бесконечности) и операторами $S, T S T^{-1}, T^{2} S T^{-2}, \ldots, T^{n} S T^{-n}$, которые и соответствуют обходам вокруг $\zeta^{i}$. С другой стороны, $T^{i} S T^{-i}$ - результат сопряжения отражения относительно $\mathscr{O}$ с помощью подкрутки на $\mathscr{O}(i)$ - не что иное, как отражение относительно $\mathscr{O}(i)$.

Следствием этого предложения является

Teорема 1.7 .3 (cp. [15]). Набор $\mathbf{E}=\langle\mathscr{O}, \ldots, \mathscr{O}(n)\rangle$ квазикатцев. Проективное пространство $\mathbb{P}^{n}$ квазикатцево.

Пусть $X$ - нечетномерная квадрика $Q_{n}, \quad \zeta$ - первообразный корень степени $\operatorname{ind}\left(Q_{n}\right)=n$ из 1 . На $Q_{n}$ существует исключительньй набор $\mathbf{E}=\langle S \otimes \mathscr{O}(-1), \mathscr{O}, \ldots$ $\ldots, \mathscr{O}(n)\rangle$, где $S$ - спинорное расслоение (см. [18]).

Обозначим через $[n]$ накрытие Куммера:

$$
[n]: \mathbf{G}_{\mathbf{m}} \stackrel{n}{\rightarrow} \mathbf{G}_{\mathbf{m}}, \quad z \mapsto z^{n} .
$$

ПРЕДЛОЖЕНИЕ 1.7.4. Локальная система $\mathscr{L}_{\mathbf{E},\left\langle 0,1, \zeta, \zeta^{2}, \ldots, \zeta^{n-1}\right\rangle}$ изоморфна $[n]^{*} \mathscr{A} \mathscr{L}_{R R}(Q)$.

ДокАЗАТЕЛЬСТво. Так же, как и вьше, монодромия локальной системы $[n]^{*} \mathscr{A} \mathscr{L}_{R R}(Q)$ задается ограничением представления $\phi_{\mathbf{E}, \mathbf{x}}$ на нормальную подгруппу индекса $n$. Она порождается оператором $T^{n}$ и операторами $S, T S T^{-1}$, $T^{2} S T^{-2}, \ldots, T^{n-1} S T^{n-1}$, которые соответствуют обходам вокруг $\zeta^{i}$, т.е. отражениям относительно $\mathscr{O}(i)$.

В предложении 1.6.9 отражение относительно спинорного расслоения определено как (левое) частное оператора подкрутки на канонический класс и оператора, равного последовательному произведению отражений относительно прочих элементов исключительного набора. Но при таком соответствии отражения относительно прочих элементов исключительного набора переходят в локальные монодромии вокруг корней из единицы, а подкрутка на канонический класс переходит в 
локальную монодромию вокруг бесконечности. Следовательно, отражение относительно спинорного расслоения соответствует локальной монодромии вокруг 0.

Таким образом, справедлива

ТЕОРема 1.7.5. Набор $\mathbf{E}=\langle S \otimes \mathscr{O}(-1), \mathscr{O}, \ldots, \mathscr{O}(n)\rangle$ квазикатиев. Нечетномерная квадрика $Q_{n}$ квазикатцева.

\section{§ 2. $D$-модули}

2.1. Гипергеометрические дифференциальные операторы. Все определения и утверждения этого пункта взяты из [22].

Рассмотрим гипергеометрические $D$-модули на одномерном торе $\mathbf{G}_{\mathbf{m}}=$ $\operatorname{Spec} \mathbb{C}\left[z, z^{-1}\right]$. Через $\mathscr{D}$ обозначим алгебру дифференциальных операторов на $\mathbf{G}_{\mathbf{m}}$, через $D$ - дифференциальный оператор $z \frac{\partial}{\partial z}$. Имеем $\mathscr{D}=\mathbb{C}\left[z, z^{-1}, D\right]$.

ОПРЕДЕЛЕНИЕ 2.1.1 [22, п. 3.1]. Пусть $n$ и $m$ - пара неотрицательных целых чисел, $P$ и $Q$ - многочлены степеней $n$ и $m$ соответственно. Определим гипергеометрический оператор типа $(n, m)$ :

$$
\operatorname{Hyp}(P, Q)=P(D)-z Q(D)
$$

и гипергеометрический D-модуль:

$$
\mathscr{H}(P, Q) \stackrel{\text { def }}{=} \mathscr{D} / \mathscr{D} \operatorname{Hyp}(P, Q)
$$

Если $P(t)=p \prod_{i}\left(t-a_{i}\right), Q(t)=q \prod_{j}\left(t-b_{j}\right), \lambda=p / q$, запишем

$$
\mathscr{H}_{\lambda}\left(a_{i}, b_{j}\right) \stackrel{\text { def }}{=} \mathscr{D} / \mathscr{D}\left(\lambda \prod_{i}\left(D-a_{i}\right)-z \prod_{j}\left(D-b_{j}\right)\right) .
$$

ПРЕДЛОЖЕНИЕ 2.1.2 [22, п. 3.2.1]. $\mathscr{H}_{\lambda}\left(a_{i}, b_{j}\right)$ - неприводимый $D$-модуль на $\mathbf{G}_{\mathbf{m}}$ тогда и только тогда, когда $P$ и $Q$ не имеют общих нулей по $\bmod \mathbb{Z}$.

ПРЕДЛОЖЕНИЕ 2.1.3 [22, П. 2.11.9]. Пусть $\mathscr{H}_{\lambda}\left(a_{i}, b_{j}\right)$ - неприводимы й гипергеометрический $D$-модуль. Eсли сard $\left\{a_{i}\right\} \neq \operatorname{card}\left\{b_{j}\right\}$, mо $\mathscr{H}_{\lambda}\left(a_{i}, b_{j}\right)$ гладок на $\mathbf{G}_{\mathbf{m}}$. Eсли $\operatorname{card}\left\{a_{i}\right\}=\operatorname{card}\left\{b_{j}\right\}$, положим $U=\mathbf{G}_{\mathbf{m}} \backslash\{\lambda\}, j: U \rightarrow \mathbf{G}_{\mathbf{m}}-$ соответствующее вложсение. Тогда $\mathscr{H}_{\lambda}\left(a_{i}, b_{j}\right)$ гладок на $U, \mathscr{H}_{\lambda}\left(a_{i}, b_{j}\right)=$ $j_{! *} j^{*} \mathscr{H}_{\lambda}\left(a_{i}, b_{j}\right)$, и локальная монодромия его решений вокруг $\lambda$ является псевдоотражсением.

ПРЕДЛОЖЕНИЕ 2.1.4 [22, П. 3.2(1)]. Пусть $\lambda$ фиксировано. Пусть $\mathscr{H}_{\lambda}\left(a_{i}\right.$, $\left.b_{j}\right)=\mathscr{H}(P, Q)$ - неприводимый $D$-модуль на $\mathbf{G}_{\mathbf{m}}$. Тогда класс изоморфизма $\mathscr{H}_{\lambda}\left(a_{i}, b_{j}\right)$ зависит только от наборов $a_{i} \bmod \mathbb{Z} u b_{j} \bmod \mathbb{Z}$. 
ПРЕДЛОЖЕНИЕ 2.1 .5 [22, П. 3.2 .2 (iii)]. Пусть $\mathscr{H}_{\lambda}\left(a_{i}, b_{j}\right)=\mathscr{H}(P, Q)-$ нeприводимый $D$-модуль на $\mathbf{G}_{\mathbf{m}}$ mипа $(n, m)$. Тогда если $n \geqslant m$ (соответственно, $m \geqslant n)$, то собственные числа локальной монодромии его решений в регулярной особой точке 0 - это $\exp (2 \pi i a)_{P(a)=0}$ (соответственно, собственные числа локальной монодромии в регулярной особой точке $\infty$ - это $\left.\exp (2 \pi i b)_{Q(b)=0}\right)$, и каждому собственному значению локальной монодромии в 0 (соответственно, в $\infty$ ) соответствует единственная жорданова клеmкa.

ПРЕДлОЖЕНИЕ 2.1.6 [22, п. 3.3]. Класс изоморфизма $\mathscr{H}_{\lambda}\left(a_{i}, b_{j}\right)$ определяет его тип $(n, m)$, наборы с кратностями $\left\{a_{i} \bmod \mathbb{Z}\right\},\left\{b_{j} \bmod \mathbb{Z}\right\}$ и, в случае $n=m$, скаляр $\lambda$.

ПРЕДЛОЖЕНИЕ 2.1 .7 (см. [22, п. 3.5.4], ср. предложение 1.2.3). Пусть $\mathscr{F}, \mathscr{G}-$ две неприводимые локальные системь на $\left(\mathbf{G}_{\mathbf{m}} \backslash\{\lambda\}\right)^{\text {an }}$ одного ранга $n \geqslant 1$. Предположим, что:

і) локальные монодромии обеих систем в точке $\lambda$ являются псевдоотражениями;

ii) характеристические многочлены локальных монодромий систем $\mathscr{F}$ и $\mathscr{G}$ в 0 совпадают;

iii) характеристические многочлены локальных монодромий систем $\mathscr{F} и$ $\mathscr{G}$ в $\infty$ совпадают.

Тогда существует изоморфизм $\mathscr{F} \cong \mathscr{G}$.

Введем операцию ${ }^{\text {red }}$ на гипергеометрических $D$-модулях. Пусть $\mathscr{H}_{\lambda}\left(a_{i}, b_{j}\right)$ приводимый $D$-модуль, т.е. такой, что множества $\left\{a_{i} \bmod \mathbb{Z}\right\}$ и $\left\{b_{j} \bmod \mathbb{Z}\right\}$ пересекаются. Пусть $\left\{a_{i^{\prime}}\right\},\left\{b_{j^{\prime}}\right\}$ - наборы, полученные из $\left\{a_{i}\right\},\left\{b_{j}\right\}$ выбрасыванием обших по $\bmod \mathbb{Z}$ элементов. Тогда по определению $\mathscr{H}_{\lambda}^{\mathrm{red}}\left(a_{i}, b_{j}\right)=\mathscr{H}_{\lambda}\left(a_{i^{\prime}}, b_{j^{\prime}}\right)$.

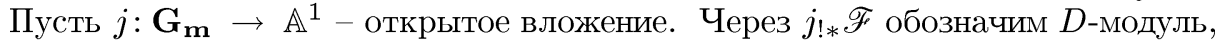
являющийся образом морфизма

$$
j_{!} \mathscr{F} \rightarrow j_{*} \mathscr{F}
$$

( "среднее продолжение"). Через FТ обозначим преобразование Фурье $D$-модулей на $\mathbb{A}^{1}$. Как обычно, если $\mathscr{F}=\mathscr{D} / \mathscr{D}\left(\sum f_{i}(x) \partial^{i}\right)$, то FT $\mathscr{F}=\mathscr{D} / \mathscr{D}\left(\sum f_{i}(\partial)(-x)^{i}\right)$.

Теорема 2.1.8 [22, п. 6.4.2]. Пусть $d$ - иелое число. Рассмотрим неприводимьй гипергеометрический $D$-модуль типа $(n, m)$

$$
H=\mathscr{H}_{\lambda}\left(a_{i}, b_{j}\right)=\mathscr{H}(P, Q) .
$$

Тогда имеются изоморфизмы неприводимых $D$-модулей на $\mathbb{A}^{1}$ :
1) $\mathrm{FT}\left(j_{! *}[d]^{*} \mathscr{H}_{\lambda}\left(a_{i}, b_{j}\right)\right) \cong j_{! *}[d]^{*} \mathscr{H}_{(-1)^{n+m} \frac{(-d)^{d}}{\lambda}}^{\text {red }}\left(1 / d, 2 / d, \ldots, d / d,-b_{j} ;-a_{i}\right)$;
2) $j_{! *}[d]^{*} \mathscr{H}_{\lambda}\left(a_{i}, b_{j}\right) \cong \mathrm{FT}\left(j_{! *}[d]^{*} \mathscr{H}_{(-1)^{n+m} \frac{(-d) d}{\lambda}}^{\text {red }}\left(1 / d, 2 / d, \ldots, d / d,-b_{j} ;-a_{i}\right)\right)$ :
$D-\bmod \left(\mathbf{G}_{\mathbf{m}}\right) \stackrel{j_{! *}}{\longrightarrow} D-\bmod \left(\mathbb{A}^{1}\right) \stackrel{\mathrm{FT}}{\longrightarrow} D-\bmod \left(\mathbb{A}^{1}\right) \stackrel{j_{! *}}{\longleftarrow} D-\bmod \left(\mathbf{G}_{\mathbf{m}}\right)$
$[d]^{*} \uparrow$
$D-\bmod \left(\mathbf{G}_{\mathbf{m}}\right)$

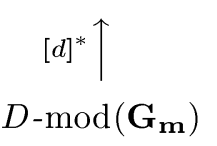




\section{2. Считающий $D$-модуль и антиканонический $D$-модуль Римана--} Poxa.

Пусть $F$ - многообразие Фано, являюшееся полным пересечением гиперповерхностей степеней $d_{j}, j=1,2, \ldots, J$, в $\mathbb{P}^{n+1}$. Для единообразия будем полагать, что $\operatorname{dim} F \geqslant 3$ и все $d_{j}>1$.

Пусть $d=n+2-\sum d_{j}$ - индекс $F, d^{\prime}=\sum d_{j}-J$. Имеем $\operatorname{dim} F=n+1-J$ и $\operatorname{dim} F+1=d+d^{\prime}$.

В п. 2.1 мы связали с таким многообразием "антиканоническую локальную систему Римана-Роха". Это неприводимая локальная система на $\mathbf{G}_{\mathbf{m}} \backslash\{1\}$, определяемая условиями на свои локальные монодромии $M_{z}, z=0,1, \infty$ :

i) $\operatorname{det}\left(1-t M_{0}\right)=\frac{1-t^{d}}{1-t} \prod_{j=1}^{J} \frac{1-t^{d_{j}}}{1-t}$

ii) $\operatorname{det}\left(1-t M_{\infty}\right)=(1-t)^{\operatorname{dim} F}$;

iii) локальная монодромия вокруг 1 - псевдоотражение.

ОПРЕДЕЛЕНИЕ 2.2.1. Пусть $\lambda \in \mathbf{G}_{\mathbf{m}}(\mathbb{C})$. Положим

$$
\begin{aligned}
\operatorname{Hyp}_{\lambda, R R}^{a}(F)= & \lambda\left(D-\frac{1}{d}\right)\left(D-\frac{2}{d}\right) \ldots\left(D-\frac{d-1}{d}\right) \\
& \times \prod_{j=1}^{J}\left[\left(D-\frac{1}{d_{j}}\right)\left(D-\frac{2}{d_{j}}\right) \ldots\left(D-\frac{d_{j}-1}{d_{j}}\right)\right]-z D^{\operatorname{dim} F} .
\end{aligned}
$$

$D$-модуль на торе вида $\mathscr{D} / \mathscr{D} \mathrm{Hyp}_{\lambda, R R}^{a}(F)$ (зависящий от параметра $\lambda$ ) назовем антиканоническим $D$-модулем Римана-Роха многообразия $F$ и обозначим $\mathscr{H}_{\lambda, R R}^{a}(F)$.

Ясно, что антиканонический $D$-модуль Римана-Роха $\mathscr{H}_{\lambda, R R}^{a}(F)$ получен из антиканонического $D$-модуля Римана-Роха $\mathscr{H}_{1, R R}^{a}(F)$ обратным образом при гомотетии $z \mapsto \frac{1}{\lambda} z$.

ПРЕДЛОЖЕНИЕ 2.2.2. Антиканонический $D$-модуль $\mathscr{H}_{\lambda, R R}^{a}(F)$ неприводим. Монодромия $D$-модуля $\mathscr{H}_{1, R R}^{a}(V)$ совпадает с монодромией антиканонической локальной системы Римана-Роха.

ДокАЗАТЕльство. Неприводимость следует из предложения 2.1.2. Утверждение о монодромии является следствием предложений 2.1.3, 2.1.5 и 2.1.7.

Рассмотрим (ср. [15]) дифференщиальное уравнение на $\mathbf{G}_{\mathbf{m}}$ ранга $\operatorname{dim} F+1=d+$ $\sum d_{j}-J=n+2-J$ следуюшего вида:

$$
D \zeta(z)=\zeta(z) M(z)=\zeta(z)\left(\begin{array}{cccccccccc}
0 & 0 & \ldots & * & 0 & \ldots & * & \ldots & & \\
1 & 0 & 0 & \ldots & * & 0 & \ldots & * & \ldots & \\
0 & 1 & 0 & 0 & \ldots & * & 0 & \ldots & * & \ldots \\
\ldots & \ldots & \ldots & \ldots & 0 & 0 & 0 & \ldots & \ldots & \\
\ldots & \ldots & \ldots & & & & \ldots & & & \\
0 & 0 & 0 & 0 & & & & & 1 & \ldots
\end{array}\right)
$$

здесь $\zeta=\left(\zeta_{0}, \zeta_{1}, \ldots, \zeta_{\operatorname{dim} F}\right) ; m_{k, l}=0$, если $k \neq l+1-i d$ ни для какого $i \in \mathbb{Z}$, $i \geqslant 0 ; m_{l+1, l}=1$; для $i \in \mathbb{Z}, i>0$, имеем $m_{l+1-i d, l}=\frac{1}{\operatorname{deg}(F)} \cdot z^{i d} \cdot i \cdot$ [число 
рациональных кривых степени $i$, проходящих через $H^{l+1-i d}$ и $H^{\operatorname{dim} F-l}$ ) (здесь $H-$ образующая в $H^{2}(F)$, т.е. класс гиперплоского сечения).

Нумерация строк и столбцов начинается с 0. В частности, в нулевой строчке первый нетривиальный элемент расположен в $(d-1)$-м столбце; число элементов в первой ненулевой диагонали составляет $\sum d_{j}-J+1$. Однако соответствующий объект должен быть определен более точно.

Перейдем к диффференциальному уравнению $(\operatorname{dim} F+1)$-го порядка, полученному из системы (2.2.1) выбором $\zeta_{0}$ в качестве циклического вектора.

Далее мы будем полагать, что $d>\frac{\operatorname{dim} F}{2}+1\left(\right.$ следовательно, $\left.d^{\prime}<\frac{\operatorname{dim} F}{2}\right)$ и в системе (2.2.1) присутствуют только числа прямых.

Положим $D^{\prime}=D+d$ и введем обозначения для чисел прямых:

$$
a_{0}=\frac{\operatorname{deg}(F)}{z^{d}} m_{0, d-1}, \quad a_{1}=\frac{\operatorname{deg}(F)}{z^{d}} m_{1, d}, \quad \ldots
$$

Последовательно найдем результаты применения степеней оператора $D$ к $\zeta_{0}$ и получим выражение

$$
\left[\lambda_{0} D^{\operatorname{dim} F+1}-z^{d}\left(a_{d^{\prime}} D^{d^{\prime}}+a_{d^{\prime}-1} D^{d^{\prime}-1} D^{\prime}+\cdots+a_{0}\left(D^{\prime}\right)^{d^{\prime}}\right)\right]\left(\zeta_{0}\right)=0
$$

с некоторой константой $\lambda_{0}$ (не будем вычислять ее точное значение).

Дифференциальный оператор в левой части этого равенства не является гипергеометрическим.

ОПРЕДЕЛЕНИЕ 2.2.3. Дифференщиальньй оператор $\operatorname{Hyp}_{\lambda, c}(F)$ (зависящий от параметра $\lambda$ ) на торе $\mathbf{G}_{\mathbf{m}}$, задаваемьй формулой

$$
\operatorname{Hyp}_{\lambda, c}(F)=\lambda D^{\operatorname{dim} F+1}-z\left(a_{d^{\prime}} D^{d^{\prime}}+a_{d^{\prime}-1}(D)^{d^{\prime}-1}(D+1)+\cdots+a_{0}(D+1)^{d^{\prime}}\right),
$$

назовем считающим дифференциальным.м оператором. D-модуль

$$
\mathscr{H}_{\lambda, c}=\mathscr{D} / \mathscr{D} \operatorname{Hyp}_{\lambda, c}(F)
$$

на торе $\mathbf{G}_{\mathbf{m}}$ назовем считающим $D$-модулем.

Отсюда видим, что оператор, стоящий в левой части (2.2.2), является $d$-куммеровым подъемом оператора $\mathscr{H}_{\lambda, c}$ для некоторого $\lambda$.

Необходимо выяснить, как считающий $D$-модуль связан с антиканоническим $D$-модулем Римана-Роха.

ТЕОРема 2.2.4. Преобразование Фурье среднего продолжения $d$-куммерова подвема (всякого) считающего модуля изоморфно среднему продолжснию d-куммерова подвема (некоторого) антиканонического модуля Римана-Роха:

$$
\forall \lambda \quad \exists \lambda^{\prime}: \quad \mathrm{FT} j_{! *}[d]^{*} \mathscr{H}_{\lambda, c}(F) \cong j_{! *}[d]^{*} \mathscr{H}_{\lambda^{\prime}, R R}^{a}(F)
$$

(см. диаграмму в теореме 2.1.8). 
ДокАЗАТЕЛЬСтво. Согласно теореме 2.1.8 достаточно показать, что корни многочленов

$$
\left(a_{d^{\prime}} D^{d^{\prime}}+a_{d^{\prime}-1} D^{d^{\prime}-1}(D+1)+\cdots+a_{0}(D+1)^{d^{\prime}}\right)
$$

и

$$
\prod_{j=1}^{J}\left(D+\frac{1}{d_{j}}\right) \ldots\left(D+\frac{d_{j}-1}{d_{j}}\right)
$$

совпадают по $\bmod \mathbb{Z}$.

Обозначим через $F(t)$ производящий многочлен последовательности чисел $a_{i}$ :

$$
F(t)=a_{0}+a_{1} t+\cdots+a_{d^{\prime}} t^{d^{\prime}}
$$

ЗАМЕчАниЕ 2.2.5. Очевидно, что если $\left\{r_{i} \mid i=1, \ldots, d^{\prime}\right\}$ - неединичные корни $F(t)$, то корни многочлена (2.2.3) - это $\left\{\frac{r_{i}}{1-r_{i}}\right\}$.

Следуюший шаг - вычисление многочлена $F(t)$ (cp. с вычислением в [2]).

2.3. Клетки Шуберта. Пусть $J=1$, а $G=G(2, n+2)$ - грассманиан 2 -плоскостей в $(n+2)$-мерном пространстве $V$ и $S$ - универсальное подрасслоение. Фиксируем полный флаг $F_{0} \subset F_{1} \subset \cdots \subset F_{n+2}$ в $V$. Тогда по определению $\sigma_{i, k}-$ когомологический класс в $G$ (замкнутой) подсхемы точек, отвечающих плоскостям $P$ таким, что

$$
\begin{gathered}
\operatorname{dim} P \cap F_{n+1-i} \geqslant 1, \\
P \subset F_{n+2-k} .
\end{gathered}
$$

Положим $\sigma_{i} \stackrel{\text { def }}{=} \sigma_{i, 0}$. Имеем

$$
d_{1}^{2} F(t)=\sum_{i=0}^{d_{1}-1}\left[\left.c_{d_{1}+1}\left(\operatorname{Sym}^{d_{1}} S^{*}\right) \cdot \sigma_{n-d_{1}+i} \cdot \sigma_{n-i-1}\right|_{\text {фунд. класс G }}\right] t^{i} .
$$

Действительно, правая часть выражения (2.2.5) - это число плоскостей в $V$, лежаших на конусе $F$ и пересекаюших обшие линейные подпространства размерностей $d_{1}+1-i$ и $i+2$, или число прямых, лежащих на $F$ и пересекающих проективные подпространства $\mathbb{P}(V)$ размерностей $d_{1}-i$ и $i+1$. Каждое из этих подпространств высекает на $F$ соответствуюшую степень класса $H$.

Для удобства перепишем тройное произведение в правой части (2.2.5) как коэффициент, с которым класс, двойственный к третьему сомножителю, входит в разложение по клеткам Шуберта произведения первого и второго (см. [34]). 
ПРЕДЛОЖЕНИЕ 2.3.1. Имеем

$$
d_{1}^{2} F(t)=\sum_{i=0}^{d_{1}-1}\left\langle c_{d_{1}+1}\left(\operatorname{Sym}^{d_{1}} S^{*}\right) \cdot \sigma_{n-d_{1}+i} ; \sigma_{n, i+1}\right\rangle t^{i}
$$

Приведем некорректное рассуждение, которое поможет воспользоваться формулой (2.2.6), а затем сделаем его корректным.

Некорректное рассуждение. Разложим формально ("принцип расщепления") расслоение $S$ в прямую сумму двух виртуальных линейных расслоений:

$$
S=S_{1} \oplus S_{2}
$$

Положим $s_{1}=c_{1}\left(S_{1}\right), s_{2}=c_{1}\left(S_{2}\right)$. Тогда старший класс Чженя расслоения $c_{d_{1}+1}\left(\operatorname{Sym}^{d_{1}} S^{*}\right)$ равен $\prod_{i=0}^{d_{1}}\left(i s_{1}+\left(d_{1}-i\right) s_{2}\right)$. Умножим это произведение на клетки Шуберта множитель за множителем. Условимся, что

$$
\begin{aligned}
& s_{1} \cdot \sigma_{a, b}=-\sigma_{a+1, b}, \\
& s_{2} \cdot \sigma_{a, b}=-\sigma_{a, b+1} .
\end{aligned}
$$

Это согласуется с правилом Литтлвуда-Ричардсона в том, что (по крайней мере, при $a \gg b$, а только эта ситуация и рассматривается) $s_{1}+s_{2}$ действительно умножается на классы Шуберта так же, как и $c_{1}(S)$, а $s_{1} s_{2}-$ как $c_{2}(S)$.

Умножив класс $\prod_{i=0}^{d_{1}}\left(i s_{1}+\left(d_{1}-i\right) s_{2}\right)$ на “длинную строку" $\sigma_{x}, x \geqslant d_{1}-1$, и рассмотрев последовательность коэффициентов разложения по клеткам Шуберта, получим производящий многочлен в следующем виде:

$$
d_{1}^{2} \prod_{i=1}^{d_{1}-1}\left(i \tau^{-1}+\left(d_{1}-i\right) \tau\right)
$$

Степени при $\tau$ отвечает выражение

$$
l_{1}-l_{2}-x
$$

где $l_{1}$ - длина верхней строки, $l_{2}$ - длина нижней строки.

Нетрудно убедиться в том, что производящий многочлен в правой части выражения (2.2.6) образован той же самой последовательностью коэффициентов. Следовательно, $F(t)=\prod_{i=1}^{d_{1}-1}\left(i+\left(d_{1}-i\right) t\right)$.

Как сделать некорректное рассуждение корректным. Например, разбить все сомножители на пары: первый с последним, второй с предпоследним. Получившиеся произведения - симметрические многочлены от $s_{1}$ и $s_{2}$, следовательно, они выражаются через $c_{1}$ и $c_{2}$.

При $J>1$ необходимо $\operatorname{Sym}^{d_{1}}$ везде заменить на $\oplus \operatorname{Sym}^{d_{j}}$. Фундаментальный класс прямой суммы будет равен произведению фундаментальных классов слагаемых, производящий многочлен будет равен произведению производящих многочленов. 
ОКОНЧАНИЕ ДОКАЗАТЕЛЬСТВА ТЕОРЕМЫ 2.2.4. ВоспоЛЬЗУемся ЗаМечанием 2.2.5. Замена $r \mapsto \frac{r}{1-r}$ взаимно однозначно отображает множество корней многочлена $F(t)$, равное $\left\{-\frac{i}{d_{j}-i} \mid j=1, \ldots, J ; i=1, \ldots, d_{j}-1\right\}$, на $\left\{\frac{-i}{d_{j}}\right\}$.

2.4. $D$-модули Римана-Роха многообразий Калаби-Яо и Фано. В пп. 2.2-2.4 рассмотрен исключительно антиканонический $D$-модуль многообразия Фано. Определим собственно $D$-модуль Римана-Роха для полных пересечений в проективных пространствах.

ОПРЕДЕЛЕНИЕ 2.4.1. Пусть $X$ - многообразие Калаби-Яо или Фано, являющееся полным пересечением гиперповерхностей степеней $d_{j}, j=1,2, \ldots, J$, в $\mathbb{P}^{n+1}$.

Положим

$$
\operatorname{Hyp}_{\lambda, R R}(X)=\lambda \prod_{j}\left(D-\frac{1}{d_{j}}\right)\left(D-\frac{2}{d_{j}}\right) \ldots\left(D-\frac{d_{j}-1}{d_{j}}\right)-z D^{\operatorname{dim} X+1}
$$

Всякий $D$-модуль на торе вида $\mathscr{D} / \mathscr{D} \operatorname{Hyp}_{\lambda, R R}(X)$ мы будем называть $D$-модулем Римана-Роха многообразия $X$ и обозначать $\mathscr{H}_{\lambda, R R}(X)$.

Таким образом, антиканонический $D$-модуль Римана-Роха многообразия Фано $X$ совпадает с $D$-модулем Римана-Роха многообразия Калаби-Яо $V$, являюшегося общим сечением пучка $K_{X}^{-1}$ :

$$
\mathscr{H}_{\lambda, R R}^{a}(X)=\mathscr{H}_{\lambda, R R}(V)
$$

ПРеДЛОЖенИЕ 2.4.2. і) D-модули Римана-Роха многообразий Калаби-Яо имеют только регулярные особенности.

ii) D-модули Римана-Роха многообразий Фано имеют иррегулярную особенность в нуле.

ДоКАЗАТЕЛЬСТвО. Это следует из предложений 2.1.5 и 2.1.3.

ТЕОРема 2.4.3. Пусть выполняется ограничение ind $X>\frac{\operatorname{dim} X}{2}+1$. Тогда (всякий) считающий модуль многообразия $X$ изоморфен обратному образу (некоторого) модуля Римана-Роха многообразия $X$ при морфизме inv обращения координаты $z \mapsto z^{-1}$ :

$$
\begin{gathered}
\forall \lambda \quad \exists \lambda^{\prime}: \quad \mathscr{H}_{\lambda, c}(X) \cong \operatorname{inv}^{*} \mathscr{H}_{\lambda^{\prime}, R R}(X), \\
D-\bmod \left(\mathbf{G}_{\mathbf{m}}\right) \stackrel{\text { inv }^{*}}{\longrightarrow} D-\bmod \left(\mathbf{G}_{\mathbf{m}}\right) .
\end{gathered}
$$

ДокАЗАТЕльСтво. Вычисление корней многочлена (2.2.3) является доказательством этой теоремы. 


\section{§ 3. $l$-адические пучки}

Дополнительные сведения об $l$-адических пучках можно получить в [10] (также см. библиографию к этой работе). Напомним только, что конструктивные $\overline{\mathbb{Q}}_{l}$-адические пучки образуют “теорию с шестью операциями” и что всякий конструктивный $\overline{\mathbb{Q}}_{l}$-пучок $\mathscr{G}$ на схеме $X$ над конечным полем $k=\mathbb{F}_{q}$ характеристики $p$ задает следовую функцию на $X(k)$ следуюшим образом: если

$$
x: \operatorname{Spec}(k) \rightarrow X
$$

- элемент из $X(k)$, то обратный образ $x^{*} \mathscr{G}$ есть конечномерное непрерывное представление $\operatorname{Gal}(k)$ в $\overline{\mathbb{Q}}_{l}$-векторном пространстве; положим

$$
\operatorname{Tr}(\mathscr{G}, x)=\operatorname{Tr}\left(F_{k}\right)
$$

где $F_{k}$ - элемент $\operatorname{Gal}(k)$, который обратен стандартной образующей

$$
s \rightarrow s^{q}
$$

этой группы. Функцию $\operatorname{Tr}$ можно продолжить по линейности на объекты производной категории.

3.1. Приблизительное равенство. Пусть $k=\mathbb{F}_{q}$ - конечное поле характеристики $p, \mathbf{G}_{\mathbf{m}}=k\left[t, t^{-1}\right]$ - одномерньй тор над $k$ и $D_{c}^{b}\left(\mathbf{G}_{\mathbf{m}}, \overline{\mathbb{Q}}_{l}\right)$ - триангулированная категория когомологически ограниченных комплексов $\overline{\mathbb{Q}}_{l}$-адических пучков на торе $\mathbf{G}_{\mathbf{m}}$ с конструктивными когомологиями.

Пусть $\mathscr{L} \in \mathrm{Ob} D_{c}^{b}\left(\mathbf{G}_{\mathbf{m}}, \overline{\mathbb{Q}}_{l}\right)$. Через $\operatorname{Tr}_{k^{\prime}}(\mathscr{L}, \lambda)$, где $k^{\prime} / k$ - конечное расширение и $\lambda \in \mathbf{G}_{\mathbf{m}}\left(k^{\prime}\right)$, обозначим следовую функцию объекта $\mathscr{L}$ относительно $k^{\prime}$-фробениуса $F_{k^{\prime}}$. Вместо $\operatorname{Tr}_{k}(\mathscr{L}, \lambda)$ будем писать просто $\operatorname{Tr}(\mathscr{L}, \lambda)$, вместо $F_{k}-$ просто $F$.

ОПРЕДЕЛЕниЕ 3.1.1. Пусть $\mathscr{L}_{1}, \mathscr{L}_{2} \in D_{c}^{b}\left(\mathbf{G}_{\mathbf{m}}, \overline{\mathbb{Q}}_{l}\right)$. Следовые функции этих объектов приблизительно равны $\left(\operatorname{Tr} \mathscr{L}_{1} \approx \operatorname{Tr} \mathscr{L}_{2}\right)$, если найдется гомотетия $c$ : $\mathbf{G}_{\mathbf{m}} \rightarrow \mathbf{G}_{\mathbf{m}}$ такая, что для всякого конечного расширения $k^{\prime}$ поля $k$ и для всякой точки $\lambda \in \mathbf{G}_{\mathbf{m}}\left(k^{\prime}\right)$ со значением в $k^{\prime}$ справедливо равенство

$$
\operatorname{Tr}_{k^{\prime}}\left(\mathscr{L}_{2}, \lambda\right)=a \operatorname{Tr}_{k^{\prime}}\left(\mathscr{L}_{1}, c \lambda\right)+b
$$

с константами $a, b \in \overline{\mathbb{Q}}_{l}, a \neq 0$, зависяшими, вообще говоря, от $k^{\prime}$.

Напомним формулу следа Лефшеца.

ПРеДЛОЖЕнИЕ 3.1.2. Пусть $X$ - отделимая схема конечного типа над $k$; $E-\overline{\mathbb{Q}}_{l}$-конструктивный пучок на $X ; \bar{X}=X \otimes_{k} \bar{k}-$ схема, полученная из $X$ расширением основного поля; $\bar{E}$ - соответствующий пучок. Тогда

$$
\sum_{x \in X(k)} \operatorname{Tr}(E, x)=\sum_{r}(-1)^{r} \operatorname{Tr}\left(F \mid H_{c}^{r}(\bar{X}, \bar{E})\right) .
$$


ДОКАЗАТЕЛЬСТвО см. в $[28$, п. 6.13.4].

Поскольку (триангулированные) прямые образы с компактным носителем коммутируют с заменой базы, верен и относительный вариант формулы Лефшеца, которым будем пользоваться далее.

ПРЕДЛОЖЕНИЕ 3.1.3. Пусть $X \stackrel{\pi}{\rightarrow} S-$ морфизм многообразий над $k, \mathscr{E}-$ обгект $D_{c}^{b}\left(X, \overline{\mathbb{Q}}_{l}\right)$. Тогда для всякого $s \in S(k)$ имеем

$$
\sum_{x \in X_{s}(k)} \operatorname{Tr}(\mathscr{E}, x)=\operatorname{Tr}\left(R \pi_{!}(\mathscr{E}), s\right)
$$

где $X_{s}-$ слой морфизма $\pi$ над $s, R \pi_{!}$- триангулированный функтор прямого образа с компактным носителем.

СЛЕДСТВИЕ 3.1.4. Пусть $\mathscr{L}_{1}, \mathscr{L}_{2} \in \mathrm{Ob} D_{c}^{b}\left(\mathbf{G}_{\mathbf{m}}, \overline{\mathbb{Q}}_{l}\right)$. Обозначим через морфизм умножения $\mathbf{G}_{\mathbf{m}} \times \mathbf{G}_{\mathbf{m}} \rightarrow \mathbf{G}_{\mathbf{m}}$, через $\pi_{1}, \pi_{2}-$ морфизмы проекиии $\mathbf{G}_{\mathbf{m}} \times \mathbf{G}_{\mathbf{m}}$ на сомножители. Следовая функиия свертки с компактным носителем двух облектов $\mathscr{L}_{1} *$ ! $\mathscr{L}_{2} \stackrel{\text { def }}{=} R \iota_{!}\left(\pi_{1}^{*}\left(\mathscr{L}_{1}\right) \stackrel{L}{\otimes} \pi_{2}^{*}\left(\mathscr{L}_{2}\right)\right)$ равна свертке их следовьх функиии:

$$
\operatorname{Tr}\left(\mathscr{L}_{1} * ! \mathscr{L}_{2}\right)=\operatorname{Tr} \mathscr{L}_{1} * \operatorname{Tr} \mathscr{L}_{2}
$$

Свертка функиий на группе определена обычным образом:

$$
(f * g)(\lambda)=\sum_{x, y \in \mathbf{G}_{\mathbf{m}}^{2}(k), x y=\lambda} f(x) g(y) .
$$

СлЕДСТвиЕ 3.1.5. Пусть

$$
\operatorname{Tr}\left(\mathscr{L}_{1}\right) \approx \operatorname{Tr}\left(\mathscr{L}_{1}^{\prime}\right), \quad \operatorname{Tr}\left(\mathscr{L}_{2}\right) \approx \operatorname{Tr}\left(\mathscr{L}_{2}^{\prime}\right)
$$

Tогда

$$
\operatorname{Tr}\left(\mathscr{L}_{1} * ! \mathscr{L}_{2}\right) \approx \operatorname{Tr}\left(\mathscr{L}_{1}^{\prime} * ! \mathscr{L}_{2}^{\prime}\right)
$$

3.2. Гипергеометрические объекты и гипергеометрические суммы над конечными полями. Материал этого пункта взят из [22].

Через AS обозначим накрытие Артина-Шрайера,

$$
\mathrm{AS}: \mathbf{G}_{\mathbf{a}} \rightarrow \mathbf{G}_{\mathbf{a}}, \quad x \mapsto x-F(x)
$$

а через К обозначим накрытие Куммера,

$$
\mathrm{K}: \mathbf{G}_{\mathbf{m}} \rightarrow \mathbf{G}_{\mathbf{m}}, \quad x \mapsto x / F(x)
$$

Известно, что прямой образ постоянного пучка при накрытии Артина-Шрайера (соответственно, Куммера) разлагается в прямую сумму локально постоянных 
пучков ранга 1 на $\mathbf{G}_{\mathbf{a}}$ (соответственно, на $\mathbf{G}_{\mathbf{m}}$ ), каждый из которых соответствует аддитивному (соответственно, мультипликативному) характеру поля $k$ :

$$
\mathrm{AS}_{*}\left(\overline{\mathbb{Q}}_{l}\right)=\underset{\psi_{i} \in \operatorname{Hom}\left(\mathbf{G}_{\mathbf{a}}(k), \overline{\mathbb{Q}}_{l}^{*}\right)}{\mathscr{L}_{\psi_{i}}}
$$

и

$$
\mathrm{K}_{*}\left(\overline{\mathbb{Q}}_{l}\right)=\underset{\chi_{i} \in \operatorname{Hom}\left(\mathbf{G}_{\mathbf{m}}(k), \overline{\mathbb{Q}}_{l}^{*}\right)}{\mathscr{L}_{\chi_{i}}}
$$

таим образом, что

$$
\operatorname{Tr}\left(\mathscr{L}_{\psi_{i}}, \lambda\right)=\psi_{i}(\lambda)
$$

и

$$
\operatorname{Tr}\left(\mathscr{L}_{\chi_{i}}, \lambda\right)=\chi_{i}(\lambda) .
$$

Выберем $\psi$ - нетривиальный $\overline{\mathbb{Q}}_{l}$-значный аддитивньй характер поля $k$. Обозначим через $\mathscr{L}_{\psi}$ соответствующий ему пучок.

Пусть $j: \mathbf{G}_{\mathbf{m}} \rightarrow \mathbf{G}_{\mathbf{a}}$ - открытое вложение, inv $\mathbf{G}_{\mathbf{m}} \rightarrow \mathbf{G}_{\mathbf{m}}$ - морфизм обращения координаты. Через $\bar{\psi}, \bar{\chi}, \ldots$ обозначим характеры, сопряженные $\psi, \chi, \ldots$

Пусть $(n, m)$ - пара неотрицательных целых чисел. Пусть $\chi_{1}, \ldots, \chi_{n}$ и $\rho_{1}, \ldots$ $\ldots, \rho_{m}-$ наборы $\overline{\mathbb{Q}}_{l}$-значных мультипликативных характеров поля $k$.

Определим [22, п. 8.2] объект

$$
\operatorname{Hyp}\left(!, \psi ; \chi_{1}, \ldots, \chi_{n} ; \rho_{1}, \ldots, \rho_{m}\right)
$$

как свертку с компактным носителем “элементарных объектов”

$$
\operatorname{Hyp}\left(!, \psi ; \chi_{i}, \varnothing\right) \stackrel{\text { def }}{=}\left(j^{*} \mathscr{L}_{\psi}\right) \otimes \mathscr{L}_{\chi_{i}}[1]
$$

и

$$
\operatorname{Hyp}\left(!, \psi ; \varnothing, \rho_{j}\right) \stackrel{\text { def }}{=} \operatorname{inv}^{*}\left(\left(j^{*} \mathscr{L}_{\bar{\psi}}\right) \otimes \mathscr{L}_{\bar{\rho}_{j}}\right)[1] .
$$

Определим отображение сдвига

$$
\lambda: \mathbf{G}_{\mathbf{m}} \stackrel{\cdot \lambda}{\longrightarrow} \mathbf{G}_{\mathbf{m}}
$$

и сдвинутый объект

$$
\operatorname{Hyp}_{\lambda}\left(!, \psi ; \chi_{1}, \ldots, \chi_{n} ; \rho_{1}, \ldots, \rho_{m}\right)=\lambda_{*} \operatorname{Hyp}\left(!, \psi ; \chi_{1}, \ldots, \chi_{n} ; \rho_{1}, \ldots, \rho_{m}\right) .
$$

Рассмотрим случай алгебраически замкнутого поля $\bar{k}$ (см. [22, п. 8.3]). Выбрав пучок $\mathscr{L}_{\psi}$, определенный с помощью $\overline{\mathbb{Q}}_{l}$-значного аддитивного характера некоторого конечного подполя $k$ поля $\bar{k}$, и набор пучков $\mathscr{L}_{\chi}, \mathscr{L}_{\rho}$, построенных по $\overline{\mathbb{Q}}_{l}$-значным ручным характерам $\pi_{1}\left(\mathbf{G}_{\mathbf{m}} \otimes \bar{k}\right)$ и параметру сдвига $\lambda \in \mathbf{G}_{\mathbf{m}}(\bar{k})$, можно по тем же правилам, что и вьше, построить объект. Этот объект, если $\lambda$ и все $\chi, \rho$ определены над $k$, получается из объекта, определенного выше над $k$, расширением скаляров с $k$ до $\bar{k}$. 
ОПРЕДЕЛЕНИЕ 3.2.1 [22, п. 8.4.1]. Наборы $\chi_{i}$ и $\rho_{j}$ называются разделенными, если они непусты и никакой из $\chi_{i}$ не совпадает с каким-либо из $\rho_{j}$.

ТЕОРема $3.2 .2\left[22\right.$, п. 8.4.2]. Предположим, что наборы $\chi_{i}$ и $\rho_{j}$ разделены. Tогда:

i) существует (неприводимый) пучок $\mathscr{H}_{\lambda}\left(!, \psi ; \chi_{1}, \ldots, \chi_{n} ; \rho_{1}, \ldots, \rho_{m}\right)$ на $\mathbf{G}_{\mathbf{m}}$ такой, что

$$
\operatorname{Hyp}_{\lambda}\left(!, \psi ; \chi_{1}, \ldots, \chi_{n} ; \rho_{1}, \ldots, \rho_{m}\right)=\mathscr{H}_{\lambda}\left(!, \psi ; \chi_{1}, \ldots, \chi_{n} ; \rho_{1}, \ldots, \rho_{m}\right)[1]
$$

ii) если $n=m$, то $\mathscr{H}_{\lambda}\left(!, \psi ; \chi_{1}, \ldots, \chi_{n} ; \rho_{1}, \ldots, \rho_{m}\right)$ - локально постоянныи пучок ранга $п$ на $\mathbf{G}_{\mathbf{m}} \backslash\{\lambda\}$.

Группа инериии точки $\lambda$ действует через свой ручной фактор псевдоотражением. Группь инериии $I_{0}, I_{\infty}$ действуют через свои ручные факторы; соответствующие представления изоморфны соответственно

$$
\bigoplus_{\text {по различным } \chi_{i_{0}}} \chi_{i_{0}} \otimes U_{\chi_{i_{0}}}
$$

где $U_{\chi_{i_{0}}}-$ унипотентная жсорданова клетка размера, равного кратности $\chi_{i_{0}}$ в наборе $\chi_{i}, u$

$$
\bigoplus_{\text {по различным } \rho_{j_{0}}} \rho_{j_{0}} \otimes U_{\rho_{j_{0}}}
$$

где $U_{\rho_{j_{0}}}-$ унипотентная жсорданова клетка размера, равного кратности $\rho_{j_{0}}$ в наборе $\rho_{j}$.

3.3. Пучок Римана-Роха. Пусть многообразие Калаби-Яо $H\left(d_{0}, \ldots, d_{J}\right)-$ полное пересечение (общих) гиперповерхностей степеней $d_{0}, \ldots, d_{J}$ в проективном пространстве $\mathbb{P}^{d}$. Имеем $d+1=d_{0}+d_{1}+\cdots+d_{J}$.

Пусть $\chi_{i}^{(j)}, j=0, \ldots, J, i=0, \ldots, d_{j}-1,-$ все мультипликативные характеры поля $k$ порядков $d_{j}$.

Будем полагать, что

$$
p=1 \bmod d_{0} d_{1} \ldots d_{J},
$$

и, следовательно,

$$
q=1 \bmod d_{0} d_{1} \ldots d_{J}
$$

При этом условии все блоки $\chi_{i}^{(j)}$ для построения рассматриваемых гипергеометрических объектов определены над основным полем. (Это условие по сути необязательно: любой блок входит в эти конструкции вместе со своими сопряженными - но оно позволяет избежать дополнительных рассуждений о $\bar{k} / k$-формах пучков.)

ОПРЕДЕЛЕНИЕ 3.3.1. Пучком Римана-Роха $\mathscr{L}_{R R}\left(H\left(d_{0}, \ldots, d_{J}\right)\right)$ дЛя мНоГообразия $H\left(d_{0}, \ldots, d_{J}\right)$ назовем гипергеометрический объект

$$
\operatorname{Hyp}_{1}(!, \psi ; \text { все } \chi_{i}^{(j)} \text { такие, что } \chi_{i}^{(j)} \neq 1 ; \underbrace{1, \ldots, 1}_{d-J \text { раз }}) .
$$


ЗАмечание 3.3.2. Теорема 3.2.2 показьвает, что пучок Римана-Роха - некое продолжение на $\mathbf{G}_{\mathbf{m}} \mathbf{l}$-адического варианта локальной системы Римана-Роха.

ОПРеДЕЛЕниЕ 3.3.3. Неприведенным пучком Римана-Poха $\mathscr{L}_{R R}^{\text {non-red }}\left(H\left(d_{0}\right.\right.$, $\left.\left.\ldots, d_{J}\right)\right)$ для многообразия $H\left(d_{0}, \ldots, d_{J}\right)$ назовем гипергеометрический объект

$$
\operatorname{Hyp}_{1}(!, \psi ; \text { все } \chi_{i}^{(j)} ; \underbrace{1, \ldots, 1}_{d+1 \text { раз }}) \text {. }
$$

ПРЕДЛОЖЕнИЕ 3.3.4. Следовая функиия оббекта $\operatorname{Hyp}_{1}(!, \psi ; 1 ; 1)$ приблизительно равна следовой функиии пучка $\delta_{1}$, т.е. характеристической функиии точки 1 на $\mathbf{G}_{\mathbf{m}}$.

ДокАзАТЕЛьСтво. Действительно, по формуле следа Лефшеца

$$
\operatorname{Tr} \operatorname{Hyp}_{1}(!, \psi ; 1 ; 1)=\sum_{x / y=\lambda} \psi(x-y)=\sum_{y \in k^{*}} \psi((\lambda-1) y)= \begin{cases}-1, & \lambda \neq 1 \\ q-1, & \lambda=1\end{cases}
$$

СлеДСтвИЕ 3.3.5. Следовье функиии приведенного и неприведенного пучков Римана-Роха приблизительно равны.

ДокАЗАТЕльство. Последовательно свертываем неприведенньй пучок с объектами $\operatorname{Hyp}_{1}(!, \psi ; 1 ; 1)$ и применяем предложения 3.1 .5 и 3.3.4.

Через $\left[d_{j}\right]$ обозначим изогению $\mathbf{G}_{\mathbf{m}} \rightarrow \mathbf{G}_{\mathbf{m}}$ степени $d_{j}$.

ОПРЕДЕЛЕНИЕ 3.3.6. Скрученным неприведенным пучком Римана-Роха $\mathscr{L}_{R R}^{\mathrm{tw}}\left(H\left(d_{0}, \ldots, d_{J}\right)\right)$ назовем объект

$$
\operatorname{Hyp}_{1}(!, \psi ; \varnothing ; \underbrace{1, \ldots, 1}_{d+1 \text { раз }}) * !\left[d_{0}\right]_{*} \operatorname{Hyp}_{1}(!, \psi ; 1 ; \varnothing) * ! \cdots * !\left[d_{J}\right]_{*} \operatorname{Hyp}_{1}(!, \psi ; 1 ; \varnothing) .
$$

Следуюшее предложение - аналог соотношений Давенпорта-Хассе (см. [21, п. 5.6.2], [22, п. 8.7.2] и [22, п. 8.7.4]).

ПРЕДЛОЖЕНИЕ 3.3.7. Пусть $d^{\prime}-$ число, $\chi_{0}, \ldots, \chi_{d^{\prime}-1}-$ все мультипликативные характеры $k^{*}$ порядка $d^{\prime}$. Тогда оббекты $\left[d_{*}^{\prime}\right] \operatorname{Hур}_{1}(!, \psi ; 1 ; \varnothing)$ и $\operatorname{Hyp}_{1}\left(!, \psi ; \chi_{0}, \ldots, \chi_{d^{\prime}-1} ; \varnothing\right)$ можно получить один из другого композичией подкрутки на геометрически постоянный пучок ранга 1 и обратного образа при некоторой гомотетии.

СлеДСтвИЕ 3.3.8. Следовье функиии неприведенного пучка Римана-Роха и скрученного неприведенного пучка Римана-Роха приблизительно равны:

$$
\operatorname{Tr} \mathscr{L}_{R R}^{\text {non-red }}\left(H\left(d_{0}, \ldots, d_{J}\right)\right) \approx \operatorname{Tr} \mathscr{L}_{R R}^{\mathrm{tw}}\left(H\left(d_{0}, \ldots, d_{J}\right)\right) .
$$


СлеДСтвИЕ 3.3.9. Следовые функции пучка Римана-Роха и скрученного неприведенного пучка Римана-Роха приблизительно равны:

$$
\operatorname{Tr} \mathscr{L}_{R R}\left(H\left(d_{0}, \ldots, d_{J}\right)\right) \approx \operatorname{Tr} \mathscr{L}_{R R}^{\mathrm{tw}}\left(H\left(d_{0}, \ldots, d_{J}\right)\right)
$$

3.4. Зеркальное однопараметрическое семейство. Пусть $d+1=d_{0}+$ $d_{1}+\cdots+d_{J}-$ разбиение $d+1$ в сумму натуральных чисел. Рассмотрим многобразие $M\left(d_{0}, d_{1}, \ldots, d_{J}\right)$ в $\mathbf{G}_{\mathbf{m}}^{d+2}$, определяемое семейством уравнений

$$
\begin{gathered}
\prod_{j, i} y_{i}^{(j)}=\lambda, \\
y_{1}^{(0)}+\cdots+y_{d_{0}}^{(0)}-1=0, \\
y_{1}^{(1)}+\cdots+y_{d_{1}}^{(1)}-1=0, \\
\cdots \cdots \cdots \cdots \cdots \cdots \cdots \cdots+y_{d_{J}}^{(J)}-1=0 ; \\
y_{1}^{(J)}+\cdots \cdots+
\end{gathered}
$$

здесь $0 \leqslant j \leqslant J$ и $1 \leqslant i \leqslant d_{j}$.

В силу этого определения имеется морфизм

$$
m\left(d_{0}, \ldots, d_{J}\right): M\left(d_{0}, \ldots, d_{J}\right) \rightarrow \mathbf{G}_{\mathbf{m}}=k\left[\lambda, \lambda^{-1}\right] .
$$

Его слой обозначим через $M_{\lambda}\left(d_{0}, \ldots, d_{J}\right)$.

ЗАмечание 3.4.1. Семейство $M_{\lambda}\left(d_{0}, \ldots, d_{J}\right)$ - это небольшое видоизменение "пучка полных пересечений, зеркально-двойственных общему полному пересечению гиперповерхностей степеней $d_{0}, \ldots, d_{J}$, в $\mathbb{P}^{d}$ " из работы [1]. (В [1] рассматриваются компактификации наших $M_{\lambda}\left(d_{0}, \ldots, d_{J}\right)$.)

\section{5. Основные теоремы.}

Tеорема 3.5.1. Следовая функция $\operatorname{Tr}\left(\mathscr{L}_{R R}^{\mathrm{tw}}\left(H\left(d_{0}\right)\right), \lambda\right)$ скрученного неприведенного пучка Римана-Роха многообразия $H\left(d_{0}\right)$ (т.е. общего сечения антиканонического расслоения на $\mathbb{P}^{d_{0}-1}$ ) приблизительно равна следовой функиии облекта inv* $R m\left(d_{0}\right)_{!}\left(\overline{\mathbb{Q}}_{l}\right)$, т.е., согласно формуле следа Лефшеца, числу точек на $M_{\frac{1}{\lambda}}\left(d_{0}\right)$.

ДокАЗАТЕЛЬство. По формуле Лефшеца следовая функция скрученного неприведенного пучка Римана-Роха многообразия $H\left(d_{0}\right)$ равна

$$
\operatorname{Tr}\left(\mathscr{L}_{R R}^{\mathrm{tw}}\left(H\left(d_{0}\right)\right), \lambda\right)=(-1)^{d_{0}+1} \sum \psi\left(-x_{1}\right) \ldots \psi\left(-x_{d_{0}}\right) \psi(y),
$$

где сумма берется по подмножеству

$$
V=\left\{\left(x_{1}, \ldots, x_{d_{0}}, y\right) \in\left(\mathbf{G}_{\mathbf{m}}^{d_{0}} \times \mathbf{G}_{\mathbf{m}}\right)(k) \mid \frac{y^{d_{0}}}{x_{1} \ldots x_{d_{0}}}=\lambda\right\}
$$


Имеем

$$
\operatorname{Tr}\left(\mathscr{L}_{R R}^{\mathrm{tw}}\left(H\left(d_{0}\right)\right), \lambda\right)=(-1)^{d_{0}+1} \sum_{\frac{x_{1} \ldots x_{0}}{y^{d_{0}}}=\frac{1}{\lambda}} \psi(f(p)),
$$

где

$$
f(p)=-x_{1}-\cdots-x_{d_{0}}+y
$$

здесь $p$ - точка на $\mathbf{G}_{\mathbf{m}}^{d_{0}}(k) \times \mathbf{G}_{\mathbf{m}}(k)$.

Отметим, что $f$ - линейная форма и, следовательно, принимает либо все ненулевые, либо только нулевые значения на $k$-точках любой прямой, проходящей через $0 \in \mathbb{A}^{d_{0}+1} \hookleftarrow \mathbf{G}_{\mathbf{m}}^{d_{0}} \times \mathbf{G}_{\mathbf{m}}$.

Преобразуем теперь сумму в правой части (3.5.1):

$$
\sum_{\frac{x_{1} \ldots d_{0}}{y^{d_{0}}}=\frac{1}{\lambda}} \psi(f(p))=\sum_{\substack{\frac{x_{1} \ldots x_{0}}{y^{d_{0}}=\frac{1}{\lambda}} \\
f(p)=0}} \psi(f(p))+\sum_{\begin{array}{l}
\frac{x_{1} \ldots x_{0}}{y_{0}}=\frac{1}{\lambda} \\
f(p) \neq 0
\end{array}} \psi(f(p)) .
$$

Первый член, очевидно, равен

$$
(q-1)\left|M_{\frac{1}{\lambda}}\left(d_{0}\right)(k)\right|
$$

Второй член равен

$$
-\left(\left|\mathbf{G}_{\mathbf{m}}^{d_{0}-1}(k)\right|-\left|M_{\frac{1}{\lambda}}\left(d_{0}\right)(k)\right|\right) .
$$

В итоге получим

$$
\operatorname{Tr}\left(\mathscr{L}_{R R}, \lambda\right)=(-1)^{d_{0}+1}\left(q\left|M_{\frac{1}{\lambda}}\left(d_{0}\right)(k)\right|-\left|\mathbf{G}_{\mathbf{m}}^{d_{0}-1}(k)\right|\right),
$$

причем второй член в сумме не зависит от $\lambda$. Теорема доказана.

ОПРЕДЕЛЕНИЕ 3.5.2. Пусть $K$ - групповая схема над $k, \phi: \Phi \rightarrow K, \psi: \Psi \rightarrow$ $K$ - два морфизма. Их сверткой назовем морфизм-композицию

$$
\phi * \psi: \Phi \times{ }_{k} \Psi \rightarrow K \times{ }_{k} K \rightarrow K
$$

здесь второй морфизм - групповой закон.

ЛЕмма 3.5.3. Морфизм $m\left(d_{0}, \ldots, d_{J}\right)$ - это свертка морфизмов $m\left(d_{j}\right)$ :

$$
m\left(d_{0}, \ldots, d_{J}\right)=m\left(d_{0}\right) * \cdots * m\left(d_{j}\right) .
$$

Доказательство леммы очевидно.

СлЕДСТВИЕ 3.5.4. Оббект $R m\left(d_{0}, \ldots, d_{J}\right)_{!}\left(\overline{\mathbb{Q}}_{l}\right)$ изоморфен свертке оббектов $\operatorname{Rm}\left(d_{j}\right) !\left(\overline{\mathbb{Q}}_{l}\right)$ :

$$
R m\left(d_{0}, \ldots, d_{J}\right) !\left(\overline{\mathbb{Q}}_{l}\right)=*_{!} R m\left(d_{j}\right)_{!}\left(\overline{\mathbb{Q}}_{l}\right)
$$


Tеорема 3.5.5. Следовая функиия оббекта inv* $R m\left(d_{0}, \ldots, d_{J}\right)_{!}\left(\overline{\mathbb{Q}}_{l}\right)$ приблизительно равна следовой функции скрученного неприведенного пучка Римана-Poxa $\mathscr{L}_{R R}^{\mathrm{tw}}\left(H\left(d_{0}, \ldots, d_{J}\right)\right)$ :

$$
\operatorname{Trinv}^{*} \operatorname{Rm}\left(d_{0}, \ldots, d_{J}\right)_{!}\left(\overline{\mathbb{Q}}_{l}\right) \approx \mathscr{L}_{R R}^{\mathrm{tw}}\left(H\left(d_{0}, \ldots, d_{J}\right)\right) .
$$

ДокАЗАТЕЛЬСтво. По определению $\mathscr{L}_{R R}^{\mathrm{tw}}\left(H\left(d_{0}, \ldots, d_{J}\right)\right)$ есть свертка всех $\mathscr{L}_{R R}^{\mathrm{tw}}\left(H\left(d_{j}\right)\right)$. Согласно теореме 3.5 .1

$$
\operatorname{Tr} \operatorname{inv}^{*} R m\left(d_{j}\right) ! \approx \operatorname{Tr} \mathscr{L}_{R R}^{\mathrm{tw}}\left(H\left(d_{j}\right)\right)
$$

Поскольку отношение приблизительного равенства стабильно относительно свертки (следствие 3.1.5), утверждение теоремы вытекает из следствия 3.5.4.

Tеорема 3.5.6. Следовая функиия оббекта inv $^{*} R m\left(d_{0}, \ldots, d_{J}\right)_{!}\left(\overline{\mathbb{Q}}_{l}\right)$ приблизительно равна следовой функции Римана-Роха $\mathscr{L}_{R R}\left(H\left(d_{0}, \ldots, d_{J}\right)\right)$ :

$$
\operatorname{Tr}_{\operatorname{inv}}^{*} \operatorname{Rm}\left(d_{0}, \ldots, d_{J}\right) !\left(\overline{\mathbb{Q}}_{l}\right) \approx \mathscr{L}_{R R}\left(H\left(d_{0}, \ldots, d_{J}\right)\right) .
$$

ДоКАЗАТЕЛЬСТвО вытекает из теоремы 3.5.5 и утверждения следствия 3.3.9.

\section{Список литературы}

1. Batyrev V. V. Dual polyhedra and mirror symmetry for Calabi-Yau hypersurfaces in toric varieties // J. Alg. Geom. 1994. V. 3. № 3. P. 493-535.

2. Beauville A. Quantum cohomology of complete intersections // Mat. Fiz. Anal. Geom. 1995. V. 2. № 3-4. P. 384-398.

3. Batyrev V. V., Borisov L. Dual cones and mirror symmetry for generalized Calabi-Yau manifolds // Mirror Symmetry II. Studies in Advanced Mathematics. V. 1 / Eds. B.R. Greene, S.-T. Yau. American Math. Soc. International Press, 1997.

4. Batyrev V. V., Borisov L. On Calabi-Yau complete intersections. alg-geom/9412017.

5. Бейлинсон $A$. $A$. Когерентные пучки на $\mathbb{P}^{n}$ и проблемы линейной алгебры $/ /$ Функц. анализ и его прилож. 1978. Т. 12. №3. С. 68-69.

6. Бондал А. И. Представления ассоциативных алгебр и когерентные пучки // Изв. АН CCCP. 1989. T. 53. № 1. C. 25-44.

7. Bondal A., Orlov D. Reconstruction of a variety from the derived category and groups of autoequivalences. Preprint MPI/97-36 (see also alg-geom/9712029).

8. Candelas P., De la Ossa X.C., Green P.S., Parkes L. A pair of Calabi-Yau manifolds as an exactly soluble superconformal theory // Phys. Lett. B. 1991. V. 258. P. 118-126.

9. Dubrovin B. Geometry of 2D topological field theories // LNM. 1996. V. 1620. P. 120-348.

10. Ekedahl T. On the adic formalism // The Grothendieck Festschrift. Progress on Mathematics. V. 87. Basel-Boston-Berlin: Birkhäuser Verlag, 1990.

11. Givental A. Equivariant Gromov-Witten invariants // Internat. Math. Res. Notices. 1996. № 13. P. 613-663.

12. Givental A. The mirror formula for quintic threefolds // Amer. Math. Soc. Transl. Ser. 2. 1999. V. 196.

13. Golyshev V., Lunts V., Orlov D. Mirror symmetry for abelian varieties // J. Alg. Geom. 2001. V. 10. P. 433-496.

14. Griffiths Ph. et al. Topics in transcendental algebraic geometry // Annals of Math. Studies. V. 106. Princeton Univ. Press, 1984.

15. Guzzetti D. Stokes Matrices and Monodromy of the Quantum Cohomology of Projective Spaces // Comm. Math. Phys. 1999. V. 207. № 2. P. 341-383. 
16. Mirror Symmetry II. Studies in Advanced Mathematics. V. 1 / Eds. Greene B.R., Yau S.-T.. American Math. Soc. International Press, 1997.

17. Horja R.P. Hypergeometric functions and mirror symmetry in toric varieties. alg-geom/ 9912109.

18. Kapranov M. M. On the derived categories of coherent sheaves on some homogeneous spaces // Invent. Math. 1988. V. 92. P. 479-508.

19. Katz N. On the calculation of some differential Galois groups // Inv. Math. 1987. V. 87. P. $13-61$.

20. Katz N. Exponential sums over finite fields and differential equations over the complex numbers: some interactions // Bull. AMS (New Series). 1990. V. 23. № 2.

21. Katz N. Gauss Sums, Kloosterman Sums and Monodromy Groups // Annals of Math. Study. V. 116. Princeton Univ. Press, 1988.

22. Katz N. Exponential Sums and Differential Equations // Annals of Math. Study. V. 124. Princeton Univ. Press, 1990.

23. Katz N. Rigid Local Systems // Annals of Math. Study. V. 139. Princeton Univ. Press, 1996.

24. Kleiman S. Algebraic cycles and the Weil conjectures // Dix Exposés sur la Cohomologie des Schémas. Amsterdam: North-Holland, 1968. P. 359-386.

25. Kontsevich $M$. Homological algebra of mirror symmetry // Proc. Intern. Congr. Math. Zürich. 1994 / Ed. S.D. Chatterji. V. 1. Basel-Boston-Berlin: Birkhäuser Verlag, 1995. P. 120-139.

26. Looijenga E., Lunts $V$. A Lie algebra attached to a projective variety // Invent. Math. 1997. V. 129. P. 361-412.

27. Manin Yu. Problems on rational points and rational curves on algebraic varieties // Surveys of Diff. Geometry / Eds. C. C. Hsiung, S.-T. Yau. V. II. Int. Press, 1995. P. 214-245.

28. Милн Джс. Этальные когомологии. М.: Мир, 1983.

29. Orlov D. Equivalences of derived categories and K3 surfaces // J. of Math. Sciences. Plenum Publ. Corp. V. 34. Alg. geom.(5). 1997. V. 85. № 6.

30. Orlov D. On equivalences of derived categories of coherent sheaves on abelian varieties. Preprint MPI/97-49 (see also alg-geom/9712017).

31. Verdier J.-L. Catégories dérivées // Lect. Notes Math. 1977. V. 569. P. 262-311.

32. Grothendieck A., Dieudonné J. Éléments de géométrie algébrique // Publ. Math. I.H.E.S. № $11,17$.

33. Berthelot P., Grothendieck A., Illusie L. Théorie des intersections et théoreme de Riemann-Roch // Springer Lect. Notes Math. 1971. V. 225.

34. Гриффитс Ф., Харрис Дж. Принципы алгебраической геометрии. М.: Мир, 1982.

Поступило в редакцию

12.X.2000 